Aus der derm. Abteilung des Allerheiligenhospitals zu Breslau. (Primärarzt Dr. Hart tu g.)

\title{
Weitere Beiträge zur Klinik und Histologie der Folliclis.
}

Von

\author{
Dr. Arthur Alexander, \\ Sekunđärarzt.
}

(Hiezu Taf. IV-VI.)

Die Erkenntnis des eigentlichen Wesens der „Tuberculide" genannten Hautaffektionen, mit anderen Worten: Die Entscheidung der Frage, ob diese Affektionen resp. ihr hauptsächlichster Vertreter, die "Folliklis", den Toxinen des Tb.Bazillus oder diesem selbst ihre Entstehung verdanken, ist in den letzten $1 \frac{1}{2}$ Jahren in Deutschland nicht wesentlich gefördert worden. Wir selbst haben in einer im Jänner 1902 erschienenen Arbeit ${ }^{1}$ ) diese Frage ausführlich besprochen und schon damals darauf hingewiesen, daß die Annahme einer direkten bazillären Entstehung der Folliklis-Effloreszenz keineswegs ron der Hand za weisen sei. Seit dieser Zeit hat diese Lehre zwar neue Anhänger gewonnen, ist aber noch keineswegs als spruchreif, oder als entschieden nach einer der beiden Richtungen hin zu betrachten. Wir hatten in den letzten $1 \frac{1}{2}$ Jahren Gelegenheit, 7 Fälle von Folliklis zu beobachten, ${ }^{2}$ ) deren Krankengeschichten ich unter Fortlassung alles Unwesentlichen in Kürze zunächst wiedergebe.

1) D. Archiv f. klin. Med. Bd. LXXI.

2) 4 davon waren in klin. resp. poliklin. Behandlung, einen verdanken wir der Liebenswürdigkeit von Herrn Dr. Legal, Chefarzt des Krankenkauses der Landesversicherung Schlesiens, 2 entstammen der Privatpraxis von Herrn Primärarzt Dr. Harttung. 
F all I. V. Sch., aufgenom. Juli 1902 Poliklinik des Allerheiligen Hospitales. 28 Jahre alt.

Anamnese: Vater an Leberleiden gestorben, Mutter gesund, 8 Geschwister, von denen angeblich keins lungenkrank ist (jedoch besteht bei einem Bruder, den wir zu untersuchen Gelegenheit hatten, der Verdacht einer beginnenden linksseitigen Spitzenaffektion), Patientin selbst gibt mit großer Bestimmtheit an, in ihrem 15. Lebensjahre Knoten in beiden Beinen gehabt zu haben, die zirka um Weihnachten herum begannen, bis Pfingsten an Zahl zunahmen, mehr gefühlt, als gesehen wurden und auf Druck schmerzhaft waren. Bei Bettruhe bildeten sie sich anscheinend zurück, um dann am Tage wiederzukommen. Nach $1 \mathrm{jäh}$ rigem Bestehen gingen einige "auf", es entstanden Geschwüre, die mit Narbenbildung abheilten; einige blieben dauernd bestehen. Auch an den Händen hatte sie öfter Knötchen und offene Stellen. Im allgemeinen glaubt sie sich zu erinnern, daß die Knoten, wenn in Ruhe gelassen, meist von selbst zurückgegangen und nur infolge von Traumen zur Vereiterung gekommen wären. Seit dieser Zeit, also ihrem 16. Jahre, hat die Kranke dann mit längeren oder kürzeren Intervallen fortdauernd solche Knoten an Armen, Händen und Unterschenkeln gehabt, die im Anfang schmerzhaft waren, dann entweder von selbst mit Hinterlassung von braunen Flecken abheilten, oder aber aufbrachen, eiterten und im Laufe von Monaten - unter Narbenbildung sich schlossen. Von der Entstehung der Knoten gebe ich ein Beispiel für viele, über das Patientin genaue Angaben zu machen in der Lage war. Am 23. Juni fühlte sie an einer ganz circumscripten Stelle oberhalb des rechten Knies ganz schnell an Intensität zunehmende lebhafte Schmerzen, ohnedafirgendetwas ander Schmerzstellezu fühlen gewesen wäre. Aber nacheinigen Stunden schon bemerkte sie in der Tiefe einen Knoten, über dem die Haut noch normal war, seit 26. Juni ist die Stelle gerötet, zugleich verschwanden die Schmerzen. Pat. klagt sehr über Schwellungen der Beine und zuweilen auch der Hände, sowie besonders konstant über Gelenkschmerzen, sie hat häufig geschwollene Drüsen am Halse.

Status Juli 1902: Mittelgroßes, etwas anämisches Mädchen in gutem Ernährungszustande. Submaxillar- und Supraclavicular-Drüsen zeitweise bis zu Kirschgröße geschwollen, zeitweise kaum fühlbar. Die Lungen wurden dreimal zu ganz verschiedenen Zeiten (Juni 1902, Oktober 1902, Juni 1903) von zuverlässiger interner Seite und zwar jedesmal von anderen Herren, die von dem vorher erhobenen Befunde nichts wußten, untersucht: Jedesmal wurden rechts hinten oben und unterhalb des Skapularwinkels knackende Geräusche gehört: Eine in der Tiefe sitzende tuberkulöse Affektion der rechten Spitze muß daher als äußerst wahrscheinlich angenommen werden. 
Der Hautstatus war sehr wechselnd, wir geben ihn wieder, wie er sich uns im Juni 1902 bot: Oberhalb des rechten Knies fühlt man in der Tiefe eine kleine harte Stelle, über der die Haut bläulichrot verfärbt und nicht verschieblich ist. Unterhalb der rechten Patella eine talergroße, infiltrierte blänliche Stelle, an der früher kleine Knoten gesessen haben sollen. Auf der rechten Wade von der Mitte des Unterschenkels bis zur Ferse bemerkt man zahlreiche, teils gerötete, teils weililiche Narben, letztere oft mit hyperämischen Hof. Dieselben sitzen zumeist auf weicher verschieblicher Unterlage, eine sehr stark hyperämische jedoch auf einem $z w e i m a r k s t u ̈ c k g r o ß e n$, tief in die Haut hinein sich erstreckenden schmerzhaften. Knoten von bla a roter Farbe. Rechts hinten an der Wade ein bohnengroßer Knoten von dem. selben Kolorit, an der Grenze des unteren und mittleren Drittels eine flache, unregelmäßig begrenzte Ulzeration mit nicht infiltriertem Grunde. Vorderseite des linken Unterschenkels normal, an der Hinterseite, von der Grenze des oberen und mittleren Drittels an abwärts zahlreiche Narben von der eben beschriebenen Beschaffenheit. Im mittleren Drittel ein kinderhandtellergroßer Bezirk, in dessen Bereich Cutis und Subcutis besonders stark infiltriert sind und die Epidermis besonders zahlreiche Narben aufweist. Inmitten der diffusen. Infiltration ein pflaumengroßer, harter, tiefgehender Knoten. In der Gegend zwischen Malleolus ext. und Achillessehne fühlt man unter der deutlich verschiebbaren Haut einen harten länglichen circumscripten Tumor, nach Angabe der Patientin zirka 10 Tage bestehend. Am linken äußeren Fußrande, noch dem dorsum pedis zugehörend, ein erst ganz kurze $Z$ eit bestehender, erbsengroßer subkutaner Knoten, der sich im Unterhautbindegewebe umberwälzen läbt, ohnedaß man den Eindruck hat, daberirgendwo fixiert ist. (Die drei zuletzt genannten Knoten sind - cf. unten - Gegenstand der histolog. Untersuchung.) Vorderarme beiderseits, besonders an den UJnarseiten mit kleinen, völlig kreisrunden, weißlich atrophischen Stellen und Narben bedeckt, die sich besonders beim Herabhängen der Extremität von dem stark bläulichen Ton, den übrigens auch die Unterschenkel in hohem Grade zeigen, deutlich abheben. Auf dem rechten Handgelenk ein kleines, tlaches, wie eine einfache Aknepustel aussehendes Knötchen, das allerdings eine auffallend derbe Infiltration seines Grundes zeigt, ebeuso am stark geschwollenen linken Daumen (Streckseite des distalen Gelenkes) eine infiltrierte Stelle, sämtliche Finger der rechten Hand zeigen ebenfalls kleine oberflächlich sitzende blaurote Knötchen. Die Hände sind im ganzen stark cyanotisch, an mehreren Fingerrücken die bekannten kleinen Narben.

27. Juni 1902. Nachmittags erhält Pat. $1 \mathrm{mg}$ Alttuberkulin in den rechten Oberarm. Keine allgemeine oder lokale Reaktion

30. Juni 1902. Nachmittags $3 \mathrm{mg}$ Alttuberkulin in den linken Oberarm. 


$\begin{array}{rrccr}\text { 1. Juli } 2 & \text { Uhr Vorm. } & 37 \cdot 0 \\ 5 & " & " & 37 \cdot 3 \\ 10 & " & " & 38 \cdot 3 \\ 1 & " & \text { Nachm. } & 38 \cdot 6 \\ 4 & " & " & 37 \cdot 9 \\ 7 & " & " & 38 \cdot 0 \\ 10 & n & " & 37 \cdot 4 \\ \text { 2. Juli } 5 & " & \text { Morgens } & 37 \cdot 3\end{array}$

1. Juli Am linken Unterschenkel ist nach Angabe der Pat. der g r o Be oben beschriebene Knoten seit gestern Abend sohmerzhaft, außerdem eine der Narben am rechten Unterschenkel. Objektiv ist in der Tat die zuletztgenannte Narbe recht deutlich bellrotgefärbt, der Knoten linksdeutlich schmerzhaft, gerötet und weicher als vorher.

Wir werden also die allgemeine sowohl, als ach die lokale Reaktion als positiv bezeichnen müssen. In den folgenden Wochen und Monaten entstanden dann bald hier, bald dort an den Extremitäten neue Knötchen, die nach einiger Zeit ohne Narbenbildung wieder zurückgingen, zum Teil auch an den Unterschenkeln und an den Fingern - wohl infolge von Traumen - aufbrachen, und unter Bildung unregelmäfiger, Eiter sezernierender flacher Ulzerationen allmählich mit Narben abheilten. In mehreren aus solchem Eiter gewonnenen Ausstrichpräparaten gelang es uns Bazillen nachzuweisen, welche eine sehr große Säure- und Alkohol-Festigkeit zeigten und in Form und Lagerung eine sehr große $\ddot{A} h n l i c h k e i t$ mit Tb.-Bazillen hatten, jedoch möchten wir uns nicht getrauen, sie mit aller Bestimmtheit als solche anzusprechen.

Aus dem Allgemeinstatus ist noch nachzutragen, daß zuweilen Ö deme der Knöchelgegend beobachtet wurden, die bei horizontaler Lage sich zurückbildeten und daß zweimal, zuletzt im Mai 1903, die rechte Hand der Pat. geschwollen war. Objektiv war nichts nachzuweisen, als eben diese diffuse Schwellung am Handgelenk und den Metakarpalgelenken; dem Sitze nach mußten wir annehmen, daß es sich um Ergüsse in die kleinen Karpal- und Metakarpal-Gelenke handle. Im Dezember 1902 hatte Pat. in der Ulnarseite der linken Hand (KleinfingerBallen) ein 1 ängliches hartes tiefliegendes Infiltrat, welches sich nicht scharf gegen die Umgebung abgrenzte, sehr schmerzhaft war und in zirka 4 Wochen nur sehr langsam zurückging; ähnliche harte, schmerzhafte Ödeme hatte Pat. des öfteren auch an den $\mathrm{Fußgelenken.}$ Am 19. Dezember 1902 wurde daraufhin noch einmal $1 / 2 \mathrm{mg}$ A lttuber$\mathrm{k}$ ulin injiziert. Am 20. Dezember starke Kopfschmerzen, Frost und Hitze. Am 21. Früh 2 Uhr $38^{\circ} 6$, also positive Allgemeinreaktion; Lokalreaktion war diesmal nicht mit Sicherheit zu erweisen, vielleicht fiel sie aber in die Nacbt, während der die durchweg poliklin. behandelte Pat. nicht beobachtet werden konnte. 
Zum Zwecke der weiteren Aufklärung des Falles werden (selbstverständlich mit Einwilligung der Kranken) bald nach der ersten Tuberkulininjektion am 14. Juli 1902 unter Lokalanästhesie die drei erwähnten Stellen am linken Unterschenkel excidiert: Fixierung in $10 \%$ Formalin, Alkoholbärtung, Zelloidineinbettung.

a) Der Knoten am linken äußeren Fußrande wird nach langer Inzision nach Durchschneidung der Epidermis und Cutis aus dem Unterhautbindegewebe mittelst der Schere losgetrennt. Der hart anzufühlende linsengroße Knoten liegt ganz tief unter der Cutis, er ist umgeben von rel normalem Fettgewebe, ziemlich scharf von diesem sich abhebend und auf dem Durchschnitt ein offenbar infiltriertes gelblich weiß gefärbtes Gewebe zeigend, in dem Andeutungen von Knötchenbildung erkennbar sind. Nach Ausschälung des Knotens wird die Haut durch Naht geschlossen.

b) Aus dem großen harten Tumor der Wade, der im klin. Bilde sehr an einem Erythema Bazin-Knoten erinnert, wird ein keilförmiges Stück excidiert u. zw. Epidermis + Cutis + Unterbautbindegewebe. Man fühlt beim Durchschneiden, daß das Messer in der Tiefe auf harten Widerstand stößt. Makroskopisch ist nicht viel an diesem Stück zu sehen, als daß das Unterhautbindegewebe etwas induriert und von sklerosierten Strängen durchzogen erscheint. Naht, trockn. Verband.

c) Excision eines Stückes oberhalb des linken Malleolus ext. Man fühlt dort noch immer den länglichen strangförmigen Tumor: Auslösung dieses länglichen Stranges mit samt dem umgebenden Gewebe mit der Schere, Epidermis wird nicht mitgenommen. Man sieht auf dem Durchschnitt, daß der erstere aus zwei neben und parallel zu einander verlaufenden Gefäßen besteht, in der Adventitia des einen dieser Gefäße sitzt ein linsengroßes, hart sich anfühlendes Knötchen. Neben diesem länglichen Strange findet sich im Fettgewebe, von außen nicht fühlbar gewesen,

d) noch ein anderer, ebenfalls linsengroßer Knoten, der nicht so offenbar und erkennbar mit einem Gefäß in Verbindung steht. Er wird ebenfalls exstirpiert: Naht, trockener Verband.

Histologischer Befund: a) cf. Fig. 1 und 2. Zerlegung des Knotens in Serienschnitte, Färbung mit Hämatoxylin - van Gieson. sowie auf elastische Fasern nach Unna-Taenzer, resp. Weigert. (Nachfärbung mit 1\% Saffranin, die aufs wärmste zu empfehlen ist.) In den ersten Schnitten bildet das Zentrum eine durch die starke Muskularis, sowie die Anordnung des elastischen Gewebes, insbesondere die Ausbildung einer ausgesprochenen Elastica int. mit Sicherheit als solche zu erkennende Arterie, deren Adventitia mehrere Vasa vasorum aufweist. Die Intima dieser Arterie ist erheblich verdickt und das Lumen daher ein minimales, jedoch in den ersten Schnitten deutlich sichtbares. Die Arterie ist umschlossen von einer schmalen Zone relativ normalen Gewelres, an diese Zone schließt sich dann, die Arterie von allen Seiten umgebend, eine breite Schicht von tuberkulösem Gewebe, das in den ersten 
Schnitten im allgemeinen aus einer diffus verstreuten Menge epitheloider Zellen besteht, in der zahlreiche Riesenzellen verteilt sind. Daneben finden sich mehr vereinzelte in noch relativ normaler Umgebung liegende circumscripte Riesenzellen-Tuberkel. Sehr bald jedoch ändert sich das Bild: In den nächsten Schnitten begegnen wir bereits an der Peripherie ausgebreiteten Nekrosen; die größeren und kleineren Septa des Fettgewebes sind kolossal verbreitert und bestehen aus kernlosem, unfärbbarem Gewebe, dessen homogenes gleichförmiges Strukturbild nur durch vereinzelte Leukocyten resp. deren Kernreste und durch z. Teil noch Blutkörperchen enthaltende gänzlich hyalin entartete Blutgefäße unterbrochen wird (Fig. 1). Diese Veränderungen beschränken sich zunächst noch auf einen bestimmten der Peripherie der Schnitte angehörenden Abschnitt, nach dem Zentrum, das heißt der beschriebenen Arterie zu, rückt andererseits das tuberkulöse, stark riesenzellenbaltige Gewebe immer weiter vor. Bald ist die vorhin beschriebene, gewissermaßen einen Wall bildende, normale Zone durchbrochen, die Adventitia und Media fallen der Tuberkulose zum Opfer. Inzwischen hat sich auch die Intima mit dem Endothel weiter an der Wuchernng beteiligt, das Lumen der Arterie ist völlig ausgefüllt von einem aus groben bläschenförmigen Zellen und Leukocyten bestehenden P fropfe (Fig. 2), der an einzelnen Stellen sogar kleine, Blutkörperchen enthaltende Gefäße - wohl neu gebildete Kapillaren - erkennen läßt. In diesem letzten Stadium zeigen in einer gewissen Zusammenfassung der einzelnen Schnitte dieselben folgendes Bild: In bunter Abwechslung relativnormales, entzündetes und tuberkulos-nekrotisches Fettgewebe, große, perivaskuläre Infiltrate enthaltende, sehr kernreiche Bindegewebs-Septen, in ihnen zahlreiche, meist mit vielen Riesenzellen ausgestattete Tuberkel, schließlich die bereits beschriebene zentrale Arterie, deren Lumen vollständig verschlossen ist, deren Media und Adventitia von tuberkulösem Gewebe völlig durch. wachsen und zerworfen ist und die nur noch durch die Anordnung der auBerordentlich widerstandsfähigen elastischen Elementedie alte Struktur als Gefä $B$ überhaupterkennen läbt. Auch sonst finden sich überall in dem tuberkulösen Gewebe zahlreiche, zum Teil noch relativ gut erhaltene, zum Teil nur noch durch einen restierenden elastischen Ring als solche sich kennzeichnende Gefäßie.

b) ef. Fig. 5. Bei der mikroskopischen Untersuchung dieses aus der Mitte eines großen, schon lange bestehenden Tumors herausgeschnittenen Stückes verzichteten wir von vorneherein auf die SerienMethode, da wir wesentliche Aufschlüsse in Bezug auf die Entwicklung des Knotens zu erhalten nicht hoffen konnten. Um so wertvoller waren uns die Ergebnisse der Biopsie dieses Stückes in Bezug auf die Erkenntnis des Sitzes und Umfanges der Veränderungen. Elastinfärbung nach Weigert mit Nachfärbung nach van Gieson bestätigte zunächst wieder 
die klin. bereits festgestellte Tatsache, daß die Hauptveränderungen im Unterhautfettgewebe ihren Sitz haben. Letzteres ist in eine bei schwacher Vergrößerung fast homogene, die Pikrinsäure sehr stark festhaltende Masse verwandelt, in welcher sofort zabllose Hoblräume, offenbar die Reste des durch den Härtungsprozeß ausgezogenen Fettes auffallen. (Fig. 5.) Mit starken Systemen erkennt man allerdings in dem anscheinend homogenen Gewebe an manchen Stellen eine gewisse Struktur: Hyaline, mit verdiekten Wandungen ausgestattete, Blutkörperchen enthaltende Gefäße, in denen das elastische Gewebe z. T. noch als deutlicher Ring erhalten, z. T. nur noch andeutungsweise in Resten erkennbar ist, sowie vereinzelte Leukocyten und zahllose Kernbröckel, welche das Ganze wie mit einer f'einen Staubschicht bedeckt erscheinen lassen. Nur in den ganz groben, die Fettlappen trennenden Septen ist das Bindegewebs- und elastische Fasernetz noch relativ gut erhalten, ein Teil dieser Septen, besonders die nach dem Seitenrande der Schnitte und nach der Cutis zu liegenden, enthalten mehr oder weniger ausgesprochen ein aus epitheloiden und Riesenzellen bestehendendes Gewebe, welches vielfach auch die in den Septen verlaufenden größeren Gefäße angegriffen und z. T. zerstört ha.t. Man sieht einerseits zahlreiche Gefäßreste in Gestalt von kreisförmig und oval angeordneten elastischen Fasern inmitten von typischem tuberkulösem Granulationsgewebe, anderseits finden wir, wieder mit Hilfe der Elastika-Färbung, die uns die Orientierung in den ziemlich komplizierten Verhältnissen ganz außerordentlich erleichtert, alle Übergänge von der beginnenden Wucherung der Intima und Media bis zum vollständigen Verschluß dieser Gefäße durch tuberkulöse, aus epitheloiden und Riesenzellen bestehende Massen. (Fig. VI.) Ganz besonders interessanterschien uns, gerade im Hinblick a f BeobachtungenandrerAutoren, das Auftreten von Verkäsungen in diesen GefäBen (Fig. VI), weil man eben ohne Kenntnis der eben beschriebenen Stadien der Endangitis tuberculosa sehr leicht dahin gelangenkann, diese Bilder nicht für das Endstadium eines tuberkulösen Prozesseszuhalten, sondern für einfacheThromben, welche sekundär zerfallen wären. Die Grenze zwischen diesem eben beschriebenen, so mannigfach veränderten Unterhautzellgewebs und der Cutis wird gebildet vonteils diffusem teils in circumscripten Knötchen angeordnetem tuberkulösem Gewebe. Die Cutis selbst, sowie der Papillarkörper sind ebenfalls hochgradig affiziert: das elastische Gewebe ist bis auf einige ganz grobe Fasern und klumpige Schollen völlig verschwunden, das bindegewebige Gerüst teilweise erhalten und von zahIreichen vorwiegend aus epitheloiden Zellen bestehenden, jedoch auch der Riesenzellen nicht entbehrenden Tuberkeln durchzogen. Auch hier finden wir wieder die bereits beschriebenen, in verschiedenem Grade bis zur gänzlichen Strukturlosigkeit veränderten Gefäße; in den Papillen sind an mehreren Stellen langgestreckte, ziemlich regelmäßige Abgrenzung aufweisende tuberkulös e Stränge sichtbar, so daß man den Ein- 
druck hat, als ob die in den Papillen verlaufenden Gefäße tuberkulös infiltriert wären. Die Epidermis ist überall intakt, die Keratohyalinschicht. zirka vier Zellagen stark, ziemlich reichliche Leukocyten durchziehen das Rete malpighi.

c) of. Fig. 3 und 4. Der ebenfalls in Serienschnitte zerlegte „Gefäßs. strang", wie wir ihn kurz nennen wollen, besteht im Anfang zunächst nur aus einem quergetroffenen, ziemlich großen, von lockerem, gefäßhaltigem Bindegewebe umgebenen Gefäß, dessen Lumen ziemlich erhebliche Veränderungen aufweist; letztere beschränken sich in den ersten Schnitten auf eine geringe Wucherung der Intima, der an einer Stelle ein aus kernreichem Gewebe bestehender Buckel aufgelagert ist. Diese Auflagerung vergrößert sich sehr rasch, so daß sie bald einen großen Teil des Lumens ausfüllt, und wir sehen auf dem Querschnitt im Lumen des Gefäßes eine aus langgestreckten bindegewebigen Zellen und straff gewebten Fasern bestehende, geringes elastisches Gewebe enthaltende Platte, welche direkt an der einen Wand entspringt und bis zur gegenüberliegenden fast heranreichend, nur noch ein geringes Restlumen frei läßt; auch dieses wird in manchen Schnitten noch verengt durch eine ebenfalls in die Gefäßlichtung hereinragende Membran, die direkt aus der Intima entspringt und aus lockerem, welligem Bindegewebe zusammengesetzt ist. Die Wand des Gefäßes selbst hat zwar eine ziemlich stark entwickelte Media, kennzeichnet sich jedoch durch die Verteilung der elastischen Bestandteile derselben und durch den Mangel einer ausgeprägten Elastica interna, sowie durch die Weite ihres Lumens deutlich als Vene. Die zuletzt erwähnte Membran ist entweder eine Venenklappe oder ein zelliger Auswuchs der Intima, jedenfalls aber nichts pathologisches. Die zuerst beschriebene, aus straffem Bindegewebe bestehende Platte dagegen halten wir für einen organ is i e r t en $\mathbf{T}$ hrombus. Übrigens verändert die Vene ihren eben geschilderten Charakter sehr bald; von Schnitt Nr. 13 an ist das Lumen normal, die Intima allerdings noch eine Zeitlang verdickt, allmählich wird das Gefäß immer kleiner und verschwindet schließlich ganz aus den Schnitten. Es ist in seinem ganzen Verlauf' blutleer. Schon lange bevor diese Vene in den Schnitten verschwand, zeigten dieselben, von ersterer durch das bereits erwähnte Bindegewebe getrennt, ein zweites großes Gefäß, wohl ebenfalls eine Vene. Avch sie ist in ihrem gesamten Querschnitt blutleer and zusammengeklappt, der Zirkulation entzogen und zeigt keine pathologischen Erscheinungen. Diese beiden die zwei Pole des Schnittes bildenden Venen sind verbunden durch ein lockeres, von außerordentlich zahlreichen Gefäßen (Arterien, Venen und Kapillaren) durchzogenes Bindegewebe, dessen Rand der Querschnitt eines großen, mit vielen ernährenden Gefäßen ausgestatteťen Nerven einnimmt. $\mathrm{Zu}$ diesen Elementen tritt in dem Teile des Bindegewebes, welches der als zweiten beschriebenen Vene nahe liegt, ein circumscriptes, zunächst nur aus einigen lymphoiden Zellen bestehendes Infiltrat, das im weiteren Verlauf der Serie zu einem abgeschlossenen, aus epitheloiden - Riesenzellen und mono- 
nucleären Leukocyten zusammengesetzten Tuberkel (Fig. 3) sich entwickelt. Aus dem einen werden bald drei zusammenfließende, jedoch bei Elastin resp. van Gieson-Färbung von einander trennbare Knötchen; dieselben zeigen im Zentrum wie auch in der Peripherie noch deutlich erhaltene, zum Teil mit Blutkörperchen gefüllte Gefäße, in einigen Schnitten auch Bindegewebsbalken und elastische Fasern, welche man ach Form und Anordnung für Gefäßreste halten könnte. Fast zu gleicher Zeit, wie die eben erwähnten Tuberkel, erscheinen in den Schnitten auch in der Adventitia der benachbarten großen Vene, etwa entsprechend den Vasa vasorum derselben, ausgedehnte entzündliche Infiltrate: Sie haben zwar keinen so typischen Bau, wie die zuletzt beschriebenen Tuberkel, müssen jedoch infolge ihres Bestehens aus einkernigen Leukocyten und ihres Gehalts an Riesenzellen im Zusammenhange mit den übrigen Erscheinungen als tuberkulöses Granulationsgewebe angesprochen werden. $\mathrm{Zu}$ diesen Veränderungen tritt nun bei $\mathrm{Nr}$. $15 \mathrm{der}$ Serie am andern Pol des Schnittes in der Nähe der thrombosierten Vene ein neues Bild: Ein circumscriptes, zunächst nur aus lympboiden Zellen bestehendes Infiltrat, daß dann später ebenfalls zu einem sehr typischen Riesenzelleatuberkel wird. Topographisch betrachtet liegen die drei oben beschriebenen zunächst doch von einander getrennten Tuberkel-Konglomerate an Stellen, welche in den vorhergehenden Schniten durch besonders reichliche Gefäßentwicklung ausgezeichnet waren. Geht man in der Serie weiter, so findet man, im groben betrachtet, in jedem Schnitte die beiden Venen und den Nerven und zwischen ihnen das tuberkulöse Gewebe, das im weiteren Verlauf sich von den drei genan beschriebenen Punkten aus nach allen Richtungen hin ausgebreitet hat. Wie schon gesagt, findet sich dann von einem gewissen Zeitpunkt ab die erste Vene nicht mehr vor, die Schnitte bestehen dann nur noch aus der zzweiten Vene, dem Nerven und dem tuberkulösen Gewebe, dieses letztere bietet ein ziemlich buntes Bild: Circumscripte Riesenzellen-Tuberkel, daneben zellreiches diffus sich a usbreitendes, durch seinen Gebalian epitheloiden und Riesenzellen als tuberkulös sichkennzeichnendes Gewebe, Fettgewebe, dessen kolossal ver breiterte Septen a s strukturlosem, Kernbröckel und Leukocyten enthaltendem Gewebe bestehen, dazwischen hyaline, gequollene Bindegewebsfasern (nach van Gieson gelblich-rot sich färbend), wohl ebenfalls im Vorstadium ihrer Einbeziehung in die tuberkulöse Wucherung und damitihres Untergangs begriffen. Ausgesprochene Verkäsung, wie wir sie bei der visceralen Tuberkulose gewöhnlich finden, vermißten wir in allen 300 durchgesehenen Schnitten, allerdings müssen wir doch die eben beschriebene Nekros e des Fettgew ebes dieser Rubrik zurechnen.

Den Veränderungen der Gefäß $B$ e haben wir naturgemäß eine ganz besondere Beachtung gewidmet: Abgesehen von der schon ausführlich 
geschilderten organisierten Thrombose der großen Vene konnten wir in vielen Schnitten ausgedehnte, bis zum vollständigen VerschluB des Lumens führende Intima-Wucherungen der mittleren Arterien nachweisen, wir fanden ferner in anderen Schnitten perivaskaläre Infiltrationen, welche, wenn wir sie in der Serie verfolgten, schließlich durch sekundäre Intima. Wucherungen den Verschluß des Gefäßes herbeiführten; besonders häufig zeigte sich an denjenigen Venen, welche in den interlobulären Septen des Nerven verliefen, das Lumen durch kompakte, bindegewebige Massen (organisierte Thromben) mehr oder weniger verschlossen. In einem, an die große thrombosierte Vene direkt anschließenden Gefäß war das Lumen zum Teil ausgefüllt durch guirlandenförmige, ihrerseits wieder ein Lumen einschließende Wucherungen, vielleicht gleichfalls ein organisierter, nachträglich von Gefäßen durchwachsener Venen-Thrombus.

d) Das unter d) beschriebene, ca. linsengroße Knötchen zeigt die gleichen, sub $a), b$ ) c) geschilderten Veränderungen. Die Schnitte enthalten entzündliches Fettgewebe, daneben tuberkulöse Wucherungen mit diffus zerstreuten Riesenzellen, verkäste Partien und die bereits oben erwähnte, tuberkulöse $\mathrm{Ne}$ krose des Fettgewebes mit der sehr charakteristischen Homogenisierung der verbreiterten Septen. Auch die Gefäße zeigen die schon oft geschilderten Übergänge von einfacher und tuberkulöser End- und Periangitis bis zum völligen Untergange ihrer Wände inkl. des elastischen Gewebes und ihrer Einbeziehung in die allgemeine Verkäsung.

Die Untersuchung zablreicher Schnitte aus allen 4 Stücken auf Tb.-Bazillen bleibt negativ.

T i er versuch e. 10./I. 1903. Exkochleation eines ulzerierten Knotens vom Unterschenkel: Ein Teil wird von Herrn Augenarzt l)r. Land mann einem Kaninchen in beide vorderen Augenkammern, ein Teil von uns selbst einem Meerschweinchen in die Bauchhöhle geimpft. Ersteres geht 5 Wochen später an Sepsis zugrunde, das linke Auge hatte das eingebrachte Stück völlig resorbiert, das rechte hatte mit der Bildung eines Pannus reagiert; am 16. Februar stirbt auch das Meerschweinchen: Keins von beiden Tieren zeigte irgend welche Zeichen von Tuberkulose.

20./IV. 1903. Wiederum Excision eines Stückchens von einem Knoten des linken Unterschenkels, Implantierung in das Peritoneum eines Meerschweinchens. 5 Wochen später Exitus des Meerschweinchens an einer Darmaffektion. Die Reste des eingeimpften Stückes finden sich im Peritonealraum, adherent an einer Stelle des Bauchfells. Mikroskopisch besteht das Stückchen aus völlig kernlosem, homogenem Gewebe, am Rande der Schnitte zahlreiche neugebildete Bindegewebszellen und Leukocyten. Offenbar ist dies die Stelle, wo das Stückchen am Bauchfell festklebte, und von diesem aus organisiert werden sollte. Bei dem Meerschweinchen wiederum keinerlei Zeichen von Tuberkulose. 
Th e r a p e $t$ is c h wurde, wie gewöhnlich, eine konsequente As-Behandlung eingeleitet, die leider bald wegen eines Arsen-Zoster unterbrochen werden mußte; wir haben sie dann später wieder aufgenommen, ohne bis jetzt jedoch einen Erfolg davon zu sehn. Wir beschränken uns daher auf die Darreichung von Roborantien und anf allgemeine hygienische Maßnahmen, deren Befolgung der Patientin, da sie in leidlichen äuferen Verhältnissen lebt, nicht allzu schwer wird. Zur Zeit (15. Sept. 1903.) ist übrigens Patientin frei von jeglichen Manifestationen ihrer Krankheit, abgesehen natürlich von den Narben.

F all II. S. Schm., 1 Jahr alt, Allerheiligen Hospital, aufgenommen auf Station 11 den 18/X. 1901, entlassen 22./IV. 1902.

A namnese nicht zu erhalten, da das Kind aus dem Armenhause kommt.

Status: Vom Oktober 1901. Ziemlich gut genährtes Kind weiblichen Geschlechts, auf dem rechten Auge eine Hornhauttrübung. Auf den Lungen voller Schall, infantiles Atmen, keine Rasselgeräusche; Kartoffelbauch, Andeutung von Rhachitis. Auf der Gesichtshaut mehrere scharfrandige, runde weiße Narben (Mundwinkel, Wange und Ohrmuschel). In der Nabelgegend eine 5 pfennigstückgroße, ganz scharf abgesetzte Narbe mit keloidartig erhabenem Zentrum, außerdem ea. noch 20 über das ganze Abdomen verteilte kleinere Narben, teils ebenfalls scharfrandig, teils mehr allmählich in die Umgebung übergehend. Am Gesäß rechts eine papulo-krustöse Stelle, darunter eine Narbe mit etwas hyperämischem Hof, im linken Hypochondrium eine rötliche, narbige Stelle. Auf der Beugeseite des rechten Unterarms 5 Narben von verschiedener Größe und Gestalt, zum Teil keloidartig erhaben, quer gestellt; auf der Streckseite 4 alte und 4 frische Narben; letztere mit hämorrhagischem Zentrum und hyperämischer Peripherie, auf dem Handgelenk ein frisches, ganz kleines, ziemlich oberflächliches Knötchen mit hellroter Umgebung. An der Ulnar-Seite des linken Unterarmes 2 kleine Narben. Auf dem rechten Oberschenkel 5 kleine Narben, auf dem Knie 2 ca. erbsengroße krustöse, infiltrierte Stellen, die offenbar aus 3 oder 4 kleineren zusammengeflossen sind. An der Hinterseite des rechten Oberschenkels mehrere Narben von ver. schiedener Größe, desgleichen am linken Unterschenkel und linken Fußrücken je eine Narbe; Kopf, Hals, Genitalien, Rücken, Vola manus und Planta pedis frei. Rumpf sehrverdächtig auf Lichen skrofulos orum. (Mehrere gelbliche, schuppende Stellen). Die große Mehrzahl der Narben zeigt bis auf wenige vereinzelte, eine ganz gleichmäBig kreisrunde Form, als wäre die Haut hier mit einem Locheisen durchbohrt worden. 20 /X. Nachmittags. $1 / 20 \mathrm{mg}$ Alt- Tuberkulin: 21 /X. Nachmittags $39 \cdot 2^{0}$, Abends 39 Grad, 22./X. Früh 38.6, Abends 38.6, 23./X. normale Temperatur. Am Rumpfe sind die erwähnten verdächtigen Stellen als deutlicher Lichen scrofulosorum zu erkennen, die große krustöse Effloreszenz am Knie hat einen hellroten Hof. Also lokale und allgemeine Reaktion positiv. Vor der Tuberkulin-Einspritzung Excision je eines Knötchens vom Handgelenk und Knie. 
Histologischer Befund: a) Kleines Knötchen „vom Handgelenk: 10\% Formain, Alkohol, Celloidineinbettung. Die Epidermis ist im Bereich des Knötchens gewuchert, etwa doppelt bis dreimal so breit, als in der Umgebung, und von durchwandernden Leukocyten durchsetzt. Geringes Ödem der Interzellularränme. Papillarkörper ist von erweiterten, mit Blnt prall gefüllten Gefäßen durchzogen, welche von Zellinfiltraten umgeben sind, die zum größeren Teil aus mono-, zum kleineren Teil aus polynucleären Leukocyten bestehen. Haarbälge und Schweißdrüsen, sowie die gesamte mittlere und untere Cutis und das Unterhaut-Fettgewebe sind normal, nur sind die fixen Bindegewebszellen und die besonders um Schweißdrüsen und Haarbälge angeordneten Mastzellen enorm vermehrt. Schreitet man nun in der Serie gegen die Mitte des Knötchens zu weiter fort, so sieht man die Zahl der polynucleären Leukocyten in der oberen Cutis und in der Epidermis sich sehr stark vermehren, so zwar, daß schließlich in ibrer Mitte die Epidermis an einer Stelle völlig von ihnen erfüllt und zerworfen wird; ein Stadium weiter und es würde zur völligen Durchwucherung und Zerstörung der normalen Epidermis-Elemente und damit zur Entstehung einer Ulzeration kommen, ein Ereignis, welchem hier durch die Excision des Knötchens vorgebeugt wurde.

b) Großes Knötchen vom Knie: Dieselbe Härtung (Celloidin-Einbettung). Im wesentlichen dasselbe Bild. Epidermis an einer Stelle verbreitert, gewuchert, und im weiteren Verlauf der Serie zuerst von Lenkocyten durchsetzt, dann von Eiter substituiert. Papillarkörper und obere Cutis in der entsprechenden Zone in nach beiden Seiten zu allmählich abklingendem Grade infiltriert von diskreten, um die Gefäße herum angeordneten Zellhaufen, zumeist mononucleären Leukocyten und gewucherten Bindegewebszellen, die jedoch im Verlauf der Serie nach der Mitte der Effloreszenz zu ebenfalls einen mehr polynucleären Charakter annehmen. Unterhautfettgewebe normal, Schweißdrüsen und Haarbälge desgleichen, von letzteren geht die Entzündung sicherlich nicht aus; überall reichliche Nastzellen.

Verlauf: Bei rein symptomatischer, nur roborierender Bebandlung (Phosphor, Lebertran etc.) bekommt Patientin einen periproktit. AbszeB, der gespalten und tamponiert glati heilt; Entlassung im April 1902 im guten Ernährungszustande. Neue Folliclis-Knötchen nicht aufgetreten, die alten Narben unverändert. Seitdem hat sich das Kind, das inzwischen auswärts untergebracht wurde, in normaler Weise entwickelt. Neue Stellen sind seit anderthalb Jahren nicht aufgetreten. Die alten Narben bestehen nocb, ebenso eine skrofulöse Augenentzündung.

F all III. A. Sch., 2 Jahre alt, Bademeisterstochter, aufgenommen 6./III. 1903. Station 11. Allerheiligenhospital. Entlassen 28./VIII. 1903.

Anamnese: Der Ausschlag besteht ca. seit Januar, heilte ab und kam immer wieder. Mutter und 6 Geschwister gesund, Vater im Januar an Isungen- und Darmtuberkulose im Allerheiligenhospital verstorben. 
Status: Sehr schwächlich gebautes Kind in elendem und vernachlässigtem Zustand. Stark nässendes Ekzem um die Nasenlöcher und Oberlippe mit Verdickung der letzteren und oberflächlicher Narbenbildung. Rbagaden an den Mundwinkeln, Konjunktivitis und eine kleine Trübung der rechten:Cornea, Kopf und Rumpf sowie Extremitäten mit reichlichen papulo-krustösen Stellen bedeckt, die in der Cutis sitzen und zur Ulzerations-Bildung tendieren. Diese Geschwüre sind scharfrandig, ziemlich tief gehend, bis 5 Pfennig-Stück groß, dünnen Ejter sezernierend, mit den, für Folliclis charakteristischen, zum Teil recht großen und tiefen Narben abheilend. Die Excision eines dieser Geschw üre ergibt keinen irgendwie charakteristischen mikroskopischen Befund, nur einfache, entzündliche, besonders in der unteren Cutis lokalisierte Veränderungen. Auf den Unterlappen der Lungen die Reste eines, wohl früher diffus gewesenen, allgemeinen Katarrhs.

Am 23./VI. $0.2 \mathrm{mg}$ Alt-Tuberkulin: Typische allgemeine, keine lokale Reaktion.

Behandlung der Eftloreszenzen nach allgemein chirurgischen Grundsätzen, allmäbliches Abheilen der Stellen, neue treten nicht auf. Entlassung am 28./VIII. 1903.

F all IV. A. L., 23 Jahre alt, Magd. Aufgenommen in das Krankenhaus der Landesversicherung Schlesien Mitte März 1903. (Dr. Legal). Anamnese: Sehr schwer zu erheben, da Patientin recht wenig intelligent ist. Die $\mathrm{Hautkrankheitbesteht} \mathrm{angeblich} \mathrm{seit} \mathrm{dem}$ 14. Lebensjahr, die Lungenkrankheit seit etnem Jahr. Erstere äußert sich darin, dab Patientin alle Jahre, angeblich im $\mathrm{H}$ er bst, Knoten in der Haut bekommt, die dann aufbrechen, eitern und mit Narbenbildung abheilen. Mutter vor 20 Jahren gestorben, war "skrofulös". Ein Bruder mit zwei Jahren gestorben, Vater gesund.

Status: Beide obere und untere Extremitäten wie übersäet mit Narben von rundlicher Form, weißer Farbe, scharfer Abgrenzung nnd zum Teil bräunlichem Rande. Am Fußrücken, Zehen, Unterschenkel, Knien, bis hinauf zur Mitte des Oberschenkels sieht man diese Narben, ebenso an den Fingern und Handrücken, Vorderarmen, bis über den Ellenbogen hinauf nach der Mitte des Oberarms zu. Im ganzen sind die Narben an den wnteren Extremitäten größer und zahlreicher, als an den oberen (vgl. Figur 7, 8). Auf dem rechten Hand. rücken sind die Narben sehr klein und in ungeheurer Zahl vorhanden. An der Vorderseitedes unteren Drittels des linken Unterschenkels fühltmanin der Tiefeim Unterhautgeweberundliche, knötchenförmige Resistenzen unter den dortbereits vorhandenen Narben, wohl Residuen von vorher stärker ausgebildeten Knoten. In viel schwächerem Grade sind solche resistente Stellen auch an der entsprechenden Stelle des rechten Unterschenkels fühlbar. Ca. 5-6 oberflächliche papelähnliche Herde unterhalb des linken Knies. (Lichen scrophulosorum ?) Die Größe der Narken schwankt von Hirsekorn- bis ein Markstück- 
Größe, an den Unterschenkeln sind allerdings noch ganz besonders große Narben, die, auch unregelmäßig in ihrer Form, wohl auf irgend welche andere Affektionen, Traumen etc. - Patientin ist als Landarbeiterin gewöhnt, obne Strümpfe zu arbeiten - die dort bestanden haben, zurückzuführen sind.

Das ganze bietet ein auferordentlich typisches und charakteristisches Bild, das wohl trotz des Fehlens frischer Erscheinungen ganz eindeutig als Folliclis angesprochen werden muß.

L ung en: bieten allerlei unbestimmte Symptome; es wird daher a) am 25. März und $b$ ) am 31. März der Patientin je $3 \mathrm{mg}$ Alt tuberkulin eingespritzt. Höchsttemperatur im ersten Falle 38.9, im zweiten Falle 386. Allgemein-Reaktion also positiv. In der Reaktion ausgeprägtes, großblasiges, klingendes Rasseln, besonders in der rechten Spitze, sowie deutliche Dämpfung daselbst. Rechts unten steht die Lunge höher als links. Tuberkel-Bazillen werden nicht gefunden. Sichere Lungentuberkulose. Daneben bestand an einem der beschriebenen Knoten am linken Unterschenkel deutliche, hellrote Reaktion; dieselbe ist besonders bei leichtem Druck gegenüber den anderen Knoten, die ein mehr brannotes Kolorit tragen, auffallend und ganz eindeutig, sie wird auch von den betreffenden Herrn Kollegen, der dieKranke genau and mehrfachtäglich beobachtet hat, bestätigt.

In Betreff des in unserer ersten Arbeit (l, c.) erwähnten Falles V. G. (Dr. Harttung) ist noch nachzutragen, daß die Kranke bei blühendem Aussehen regelmäBig im Herbst einen Schub von FolliclisEffloreszenzen bekommen hat, dessen letzter sich nicht auf die Extremitäten beschränkt, sondern auch das Abdomen mit zahlreichen Knoten bedeckt hat. Im allgemeinen ist der Verlauf der einzelnen Effloreszenz bis zu ihrer Heilung schneller geworden, auf den Lungen ist der alte Status wenig verändert. Patientin nimmt dauernd fast Arsen und befindet sich in äußerst sorgfältiger Pflege. Tuberkulin konnte leider aus äußeren Gründen nicht gegeben werden.

Fall V. Dr. Harttung. Frl. T. M., 38 Jahr, Privatlehrerin, sehr zart, anämisch und nervös. An den Spitzen zeitweise in jahrelanger Beobachtung etwas scharfes und verlängertes Inspirium, zeitweise perkussorisch und auskultatorisch normale Verhältnisse. Pat. ist sehr schwer Untersuchungen des Körpers zugänglich zu machen, die dann immer sehr schnell ausgeführt werden müssen.

Sie kommt 1897 mit 5 kleinen L upusherden auf beiden Wangen, die kaustisch ansgiebig zerstört werden. Frühere Herde im Gesicht, die seinerzeit Jadassohn ebenso zerstört hat, sind ohne Rezidiv geblieben. Am Körper sonst nichts von tuberkulösen Erscheinungen zu entdecken; ich sehe die Pat. alle Jahre 1-2mal ; 1898 und 1899 Schmerzen in der Halswirbelsäule, die von anderer Seite als spondylitisch-kariöse Prozesse angesprochen werden. Pat. liegt ohne Erfolg Monate im Streckverband. Dann stellen sich Schmerzen in den Fingergelenken, im Knie und in der $\mathrm{H} u ̈ f t e$ ein, die als arthritisch gedeutet, auf eine entsprechende 
Therapie zurückgehen. Von Tb. bis 1903 keine Rezidive im Gesicht oder am Körper. Anfang 1903 stellt sich mir Pat. vor mit einigen kleinen Folliklisknötchen an beiden Armen, gleichzeitig wiederum Schmerzen in der rechten Hüfte, starke Abmagerung, sehr schlechtes Allgemeinbefinden. Die Gesichtslupusstellen ganz glatt, an einzelnen Operationsstellen Pigmentablagerung, Pigmentverlust in der Ungebung. Nun erst entdecke ich auch bei einer genauen Untersuchung, die endlich konzediert wird, typische Follikl isnarben, so charakteristisch, wie sie nur sein können, an den Unterschenkeln, über das Knie bis zur Hälfte des Oberschenkels hinaufreichend und an der Clnarseite beider Arme bis zum Olekranon; diese Narben sind sehr fein und wenig auffallend. Pat. will nichts rechtes von ihrer Entstehung wissen, sie habe wohl hier und da einmal Pusteln gehabt, die jedoch nicht weh getan hätten, dann aber unter einfachen Pdastern langsam geheilt seien. Sie hätte ihnen keine Bedeutung beigemessen; die jetzigen seien viel größer und hätten auch zu Anfang sehr geschmerzt. Pat. wird mit Rücksicht auf das Hüftgelenk in das Augusta-Hospital aufgenommen. Hier Injektion von Tuberkulin $1 \mathrm{mg}$ ohne lokale und allgemeine Reaktion.

Die Lungen waren übrigens zur Zeit der Aufnahme, soweit das überhaupt physikalisch festzustellen ist, vollkommen frei, zeigten auch nach den Tuberkul.-Inj. keine Veränderung. Schließlich tritt auf $3 \mathrm{mg}$ Alt-Tuberkulin allgemeine und lokale Reaktion ein, gleichzeitig reagieren alle alten $L$ upusoperationsnarben und es bildet sich ein diffuses hämorrhagisches, in weiten, handAächengroßen Flecken über Rücken und Abdomen sich ausbreitendes Exanthem, ganz der hämorrhagischen Reaktion eines enorm ausgedehnten Lichen scrophulos orum gleichend, und auch wie dieser schließlich mit schmutzigen verwaschenen Pigmentflecken abheilend.

Pat., die der Hüfte wegen in Gips gelegen hatte, wird nach zirka 6 Wochen entlassen und macht zu Hause noch eine Art Liege- und Mastkur durch. Im Juli 1903 sehe ich sie bei leidlichen Wohlbefinden wieder, von Folliklis-Eruptionen noch frische rotgeränderte Narben, keine neuen Eruptionen. Die Hüfte erheblich gebessert; die alten Lupusherde im Gesicht wieder nur als blasse Narben kenntlich, stärkere Pigmentverschiebung an diesen Stellen. Am Rücken, Abdomen und an den Obersehenkeln bräunlich schmutzige Pigmentflecken. Ordination: Landaufenthalt, As-Fe und sonstige Roborantien.

Fall VI. Dr. Harttung. Herr B., 38 Jahre, Großkaufmann, seit 10 Jahren verheiratet, gesundes Kind von 8 Jahren, angeblich immer gesund gewesen. Juni 1902 frische Gonorrhoe-Infektion, Prostatitis, suspekte Balanitis, starke Allgemeinaffektion, Nachtschweiße, große Prostration, Lungen bei Beginn seiner Erkrankung ganz frei. 6 Wochen post infektionem wird seine Lues manifest, Pat. soll eine Schmierkur beginnen, erkrankt aber akut an einer einseitigen Pleuropneumonie mit Exsudat und sehr hohen Temperaturen. Es ist nur möglich in der häuslichen Behand- 
lung eine Überstreichungskur von 48 Ü̉berstreiohungen à $3 \cdot 0 \mathrm{~g}$ zu machen, innerlich Arsen. Im Sputum keine Tub.-Baz., dann monatelang Erholung, Aufenthalt in einem schlesischen Wintersanatorium mit sichtlichem Erfolge.

Februar 1903 regelrechte Schmierknr durch Wärter, und nach S chlaß derselben unter erneuten Nachtschweißen Ausbruch eines echten aggregierten Syphilids auf dem Rücken, und einzelner, kleiner, papulöser, sehr derber Knoten auf den Armen beiderseits z. T. Unterarm, z. T. Oberarm. Während das aggregierte Syphilid gut auf Lokalbehandlung reagiert, nebmen die Armknoten an Intensitat zu und erstjetzt finde ich neben diesen Knoten typische Folliklisnarben in mäßiger Anzahl an Armen (Ulnarseite), an den Händen und anden Unterschenkeln. Nun werden auch die Knoten-Eruptionen an den Armen als Folliklis-Eruptionen manifest, und um das Bild zu vervollständigen, stellt sich unter meiner genauesten Beobachtung ein ganz typischer tiefer Knoten in der linken Wade ein: heftiger, lokalisierter Schmerz, ohne anatomisches Substrat, das erst am 2. Tage in Gestalt eines kleinen, derben Knotens fühlbar wird. Der Knoten wächst, verschmilzt mit der Oberhant und zeigt nun das ganzeindeutige Folliklisbild; nachher entwickeln sich noch $4-5$ Knoten an den Unterschenkeln mit dem üblichen torpiden Verlauf. Pat. wird zur Weiterbehandlung nach Wiesbaden geschickt, ich habe ihn seitdem nicht wieder gesehen. Er bestreitet übrigens, von diesen Knotenerkrankungen früher etwas bemerkt zu haben, auch Pickel oder Pusteln will er niemals gehabt haben.

An m.: Der Kranke ist später auch von Jadassohn gesehen und behandelt worden. J. hat sich meiner Auffassung nicht ganz anschließen können, sondern die Armeffloreszenzen für nodöse Syphilide angesprochen, die Frage nach der Natur der Knoten an den Beinen aber offen gelassen, allerdings mit dem Hinzufügen, daß es sich hier wohl um Folliklisknoten handeln $k$ önne. Ich möchte dem hinzufügen, daß doch der Verlauf und die Heilung wenigstens eines Teiles der Armknoten nach einer von $J$. eingeleiteten energischen $\mathrm{Hg}$-Kur mit Inj. mehr dem eines Folliklisknotens, als dem eines papulo-nodősem Syphilids entspricht. Denn nur ein Teil der Knoten ging auf die Hg-Behandlung die gewöhnliche Rückbildung ein, ein anderer Teil zerfiel langsam rom Zentrum aus mit flachen, spärlich sezernierenden Geschwüren und heilte unter den bekannten randpigmentierten Narben ab.

Fall VII. H. P., Agententochter, 16 Jahre alt. Aufgenommen St. 9, Allerheiligenhospital 28./XI. 1901. Entlassen 13/XII. 1902, dann von Herrn Dr. Harttung poliklin. weiter beobachtet, auch von mir öfter gesehen.

Anamnese: Gesunde Familie, früher immer gesund gewesen; seit einigen Jahren auffallende Mattigkeit, seit ca. $1 / 2$ Jahre warzenförmige Effloreszenzen an Armen und Beinen. 
Status: Ca. 12-14 etwa erbsengroße, kreisrunde, derb anzufühlende, von der Umgebung gut zu isolierende Effloreszenzen an den Handrücken, Vorderarmen, Fußrücken und Fußsohlen: Kruptionen von ${ }_{z} \mathrm{Tu}$ berculosis verrucosa cutis", auch histologisch als solche identifiziert. Excision derselben. Glatter Heilungsverlauf. Entlassung. Dann in den nächsten Monaten tuberkulöse Coxitis und Gonitis, sehr langes Liegen im Gipsverband. Während dieser Zeit, also von Juni bis Dezember 1902, fortwährende Eruptionen von bis erbsengroben in der Cutis liegenden Infiltraten, bald hier, bald dort an den Extremitäten, sehr bald mit der Haut verlötend, und dann, zur Pustulation gekommen, mit den bekannten Narben abbeilend. Seit ca. $1 / 2 \mathrm{Jahr}$ bei rel. Wohlbefinden. - Pat. befand sich immer in gutem Ernährungszustande, die Lungen sind absolut frei - allmähliche Ausheilung der Coxitis und Gonitis, auch neue Folliklis-Effloreszenzen treten nicht auf.

Aus den mitgeteilten Krankengeschichten geht wieder eine Tatsache mit unwiderleglicher Sicherheit hervor: Der Zusammenhang derFolliclis mit der Tuberkulose. Wie wir uns diesen Zusammenhang denken, darauf wollen wir weiter unten noch zurückkommen, hier möchten wir nur auf einige wichtige Punkte im klinischen Bilde die Aufmerksamkeit lenken.

Soweit wir über eine längere und genauere Beobachtungszeit verfügen konnten (Fall I), war zu bestätigen, daß das Auftreten der Knötchen zumeist in Schüben von mehreren erfolgt. $\mathrm{Zu}$ registrieren ist hier auch die auffallende Angabe (Fall IV und Fall V G.), daß die Knoten in jedem Herbst wiederkehren; ähnliche Beobachtungen machen auch $\mathrm{Hallo}$. pea $\mathbf{u}^{1}$ ) und $\mathrm{B}$ ü r e a u, ${ }^{1}$ ) sowie $\mathrm{T}$ a $n$ v et. ${ }^{2}$ ) Die ersteren berichten von ausschließlichem Auftreten im Winter, der letztere im Sommer. Ferner fiel der Patientin Fall I auf, daf die Knoten sich mit Vorliebe an Stellen bildeten, wo bereits frïher solche gesessen hatten und zurückgegangen waren. Wir konnten diese Beobachtung der Pat. zu wiederholten Malen bestätigen; die Ähnlichkeit mit der Sy philis, deren Effloreszenzen sich ja auch mit Vorliebe an Orten syphilitischer Residuen lokalisieren ( $\mathrm{Neu}$ mann, Unna), erscheint uns hier unverkennbar.

Sehr bemerkenswert sind die fortwährenden Klagen derselben Patientin über Gelenkschmerzen, Rheumatismus. Oft konnten wir keine klinisch nachweisbare Ursache für diese Beschwerden finden, einmal allerdings waren die kleinen Gelenke der rechten Hand wochenlang geschwollen. Auch bei Fall V

1) Annales de dermat. 1896. pag. 1310.

2) Zit. nach Török (Mrazeks Handbuch).

Arch, f. Dermat. u. Syph. Bd. LXX. 
sind vielfach ähnliche Beschwerden notiert. Hier, sowie bei Fall VII liegt außerdem noch eine Gelenktuberkulose vor. Mit diesen Gelenkschmerzen beschäftigt sich auch Lerrede ${ }^{1}$ ) und führt mehrere diesbezügliche Beobachtungen aus einer von Ha ury unter seiner Leitung angefertigten Dissertation an. $\mathrm{Haury}$ tand bei $50 \mathrm{zu}$ allen Gruppen gehörigen Tuberkuliden $15 \mathrm{mal}$ Gelenkschmerzen, sei es anamnestisch, sei es zur Zeit der Beobachtung; vielleicht sind sie ebenfalls mit dem Grundleiden, der Tuberkulose, in Beziehung zu bringen und entweder durch embolische Vorgänge zu erklären, oder aber als Allgemeinerscheinungev der vollzogenen Überschwemmung des Körpers mit Infektionsmaterial aufzufassen.

Die bei Fall 1 erwähnten ödematösen, schmerzhafte $\mathrm{n}$ Infiltrate $\mathrm{e}^{2}$ ) (Kleinfingerballen, Malleolargegend) sind wohl entstanden durch Zusammenfließen von mehreren sehr tief gelegenen Folliklisknötchen, welche statt nach der Cutis sich nach der Fascie oder Muskulatur zu entwickelt haben.

Ein wie uns scheint recht wichtiger Punkt verdient noch hervorgehoben zu werden: Die Folliclis ist einmal eine exquisit chronische Affektion, in deren Verlauf die anfallsfreien Perioden, in denen die Pat. nur Narben zeigen, bei weitem überwiegen und sie ist andrerseits ausgezeichnet durch die Vielgestaltigkeit ihrer Einzeleffloreszenzen, die manchmal so unbedeutend sind, daß sie rom Kranken gar nicht beobachtet und vom Untersucher sehr leicht übersehen werden. In einem solchen Stadium relativen Freiseins von frischen Erscheinungen stellten wir Fall I am 6. Eebruar 1903 in der med. Sektion der Schles. Ges. für vaterl. Kultur vor, sie hatte damals nur 2 kleine Knötchen am vierten und fünften Finger der linken Hand, von denen der eine zur Pustulation gekommen war, sowie einen Knoten am Unterschenkel, sonst nur Narben. Wir knüpften danıals folgende Bemerkungen an diese Vorstellung: "Als wir die Patientin vorgestern ansahen, erschien der eine Knoten am Finger wie eine reine folliculäre Haarbalgentzündung, die andere Stelle so, als hätte sich die Kranke die Hände erfroren. Vor einer Reihe von Jahren hätte niemand von uns in diesen Erscheinungen etwas besonderes beobachtet, weil es eben Erscheinungen sind, so einfach, so unbedeutend und so wenig den Patienten belästigend, daß sie der Beachtung

1) Les notions nouvelles sur les Tuberculoses de la peau et les Angiodermites tab. Revue prat. des malad. cutan. syph. et rén. Band I. Nr. 9. Dez. 1902.

2) Möglicherweise sind diese Infiltrate ein Analogon oder gar identisch mit dem zuerst von Kaposi beim "Lupus erythematodes diss." beschriebenen "Erysipelas perstans", welches Boek (Archiv f. Dermatol. Bd. XLII, pag. 82) als häufige Begleiterscheinung der Folliclis anpricht. 
entgehen. Gerade aus diesem Grunde wïnscht Herr Dr. H a r t tung, daß dieser typische Fall Ihnen gezejgt wird, weil zweifellcs eine Unmenge solcher Krankheitsfälle der Beobachtung entgehen, einer Beobachtung und Erkenntnis, deren Wichtigkeit (cf. histolog. Befund) auBer Erörterung stehen dürfte."

Wir brauchen hier nur darauf hinzudeuten, wie wichtig im konkreten Falle die Kenntnis dieser an und für sich so unbedeutend erscheinenden Affektion werden kann. Sie ist gewissermaßen die Projektion einer visceralen Tuberkulose auf die Haut, das heißt derjenigen Form der Tuberkulose, deren frühzeitige Erkennung wir ja heutzutage mit allen Mitteln anstreben. Wir sind eben hier im stande, sichtbare Infektionsherde, die eine ständige Quelle der Gefahr für den Allgemein-Körper bilden, von Grund aus zu zerstören; und andrerseits geben uns diese Hauteruptionen sehr oft schon zu einer Zeit, wo die inneren Organe anscheinend noch absolut gesund sind, besonders in den lungen sich keinerlei Veränderungen auffinden lassen, ein ernstes Zeichen der Gefahr; welche über dem Organjsmus schwebt. Sie ermöglichen uns so eine Prophylaxe zu allerfrühester Zeit, mit anderen Worten, sie führen uns dem Endziel aller unserer therapeutischen Bestrebungen um einen großen Schritt näher.

In mehrfacher Beziehung scheint uns dann noch Fall VII des Interesses und besonderer Erwähnung wert zu sein. Das Auftreten der Tuberkulosis verrucosa cutis in so multiplen Herden ist zunächst schon an und für sich ein höchst ungewöhnliches Ereignis, ganz besonders bemerkenswert aber erscheint uns, das hier der eruptiven Form dieser sonst ganz langsam sich entwickelnden und meist solitär bleibenden Erkrankung ein Folliklisschub folgt. Dieser Umstand legt den Gedanken nahe, daß es Übergangsformen gäbe zwischen der labileren Form der Tuberkulose, der Folliklis, und der stabileren, der Tub. verruc. cut. Vielleicht ist der bei Fall I erwähnte Knoten, der wie ein Erythem-Bazin-Knoten sich anfühlt, eine solche Übergangsform, ebenso gehören auch offenbar alle die bei Fall I von uns excidierten Stücke zu derjenigen Form der Folliclis, welche ohne zum Zerfall oder zur Resorption zu tendieren, zu einer recht langen Lebensdauer ihrer Einzel-Effloreszenzen neigt. Übrigens ist sicherlich die Kluft zwischen der Hauttuberkulose in der obigen Varietät und manchen Foliclis-Formen nicht gar so groß: Die Brücke bildet hier der histologische Befund: In beiden Fällen Tuberkel.

Soviel über die klinischen Verhältnisse. Wenden wir uns jetzt der Deutung unserer histologischen Präparate 
$\mathrm{zu}$, so werden wir von vornherein $\mathrm{zwei}$ Gruppen unterscheiden müssen. In die erste gehört Fall $I$, in die zweite Fall II und III.

Erste Gruppe. Bei der Aneinanderreihung der vielen, serienweise durchgesehenen Schnitte von Fall I und bei ihrer Zusammenfassung zu einem Ganzen werden wir uaturgemäß unser subjektives Ermessen nicht ganz ausschalten können. Haben wir doch die uns vorliegenden Veränderungen nicht in ihrer Entwicklung verfolgen können, sondern mußten uns, nachdem sie schon eine Zeitlang bestanden, auf Grund der uns zur Verfügung stehenden fertigen Bilder eine Vorstellung zu machen suchen, wie sie entstanden sein könnten.

Aber Deutungen pathologisch-histologischer Bilder sind ja schließlich immer subjektiv. Ich erinnere nur an die Diskussion über die Frage der Histogenese des Carcinoms seit $\mathrm{R}$ ib berts Untersuchungen aus neuester Zeit. Auch hier hat man mit Recht hervorgehoben, daß noch niemand einen Krebs unter dem Mikroskop habe wachsen sehen können, daß man vielmehr nur aus den zur Verfügung stehenden Einzelbildern retrospektiv sich Klarheit zu verschaffen suchen müsse, wie diese entstanden und wie sie zu einem einheitlichen Ganzen zusammenzufügen sein möchten.

Wir werden also versuchen müssen, hauptsächlich gestützt auf die Befunde der serienweise untersuchten Knoten a) und c) uns ein Bild der Entstehung der Folliclis-Effloreszenz zu machen :

Wir nehmen an, dak alle vier von uns excidierten Stïcke entstanden sind durcheinen embolischen Prozeß. Von irgend einer Stelle im Körper, aus der Lunge oder den Drüsen oder einem anderen Organe, in dem sich tuberkulöse Prozesse abspielen, sind Tuberkelbazillen in den Kreislauf gelangt und haben, in den kleinen Arterien des Unterhautfettgewebes stecken bleibend, hier zunächst eine tuberkulöse End-oder Periarteriitis erzeugt. Ob diese kleinen Arterien zufällig Vasa vasorum irgend einer großen Vene oder Arterie waren, oder Ernährungsgefäße der Fettläppchen, ist für die prinzipielle Auffassung des ganzen Vorgangs belanglos. Von dieser tuberkulösen End-und Periarteriitis aus - so glauben wir - haben sich dann die Bazillen in dem Gewebe kontinuierlich ausgebreitet und dort jene von uns beschriebenen Tuberkel mitihren mannigfachen Folgezuständen (Verkäsung, Nekrosedes 
Fettgewebes, Gefäßveränderungen) erzeugt. Danach ist für uns in Knoten a) - vergl. Figur 1 - irgend einer der Seitenäste oder ein arterielles Ernährungsgefäß der großen Arterie der ursprüngliche Sitz des Embolus gewesen; von ihm aus ist die Tuberkulose des Unterhautfettgewebes und neben anderen Gefäßveränderungen auch eine Intimawucherung der großen Arterie selbst entstanden, die schließlich, nachdem auch Adventitia und Media von außen her durchbrochen worden sind, eine vollständige Endarteritis obliterans (Figur 2) erzeugt bat.

Ännlich in Knoten $c$ ). Auch hier halten wir für das Pri-

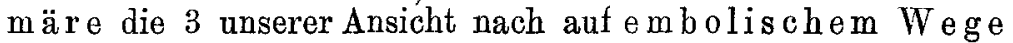
entstandenen, oben ausführlich geschilderten $\mathrm{Tub}$ erkel (cf. Figur 3). Diese sind dann weiter gewachsen, haben zu Ko mpressionen und sekundärer Thrombosierung eines Abschnittes einer größeren in ihrer Nachbarschaft verlaufenden Vene geführt, einige Ästem derselben sind mit ergriffen worden, und nach längerem Bestande ist es dann schließlich z ur Organisation der Thromben (c.. Figur 3) gekommen.

Viel weniger übersichtlich liegen die Verbältnisse bei Knoten $b$ ) und $d$ ), hier können wir eben nur sagen, daß wir einen im Unterhautfettgewebe lokalisierten, sekundär offenbar sehr stark die Gefäße (Figur 5) in Mitleidenschaft ziehenden tuberkulösen Prozeß vor uns haben, der auch auf die Cutis übergegriffen hat, und über dessen Ausgangspunkt sich wenigstens auf Grund der histologischen Bilder - nichts mehr aussagen läßt.

Wenn wir nun immer mit einer an Gewißheit grenzenden Bestimmtheit von der embolischen Entstehung der Folliclis gesprochen haben, so liegt unser $\mathrm{Hauptbew}$ is für die Richtigkeit dieser Behauptung auf rein $k l i n$ is chem Gebiete: Wir haben - wenigstens Pat. Fall I - viele Monate lang genau beobachtet, wir haben zahlreiche Knoten vor unseren Augen entstehen sehen und haben von der sehr auf sich achtenden Kranken immer und immer wieder die ganz be. stimmte Angabe erhalten, da $\beta$ sie zuerst an den betreffenden Stellen einen circumscripten Schmerz gefiihlt habe, und daß dann erst einige Tage später ein Knoten daselbst entstanden sei. Wir haben auch die Richtigkeit dieser Angabe selbst nachprüfen können: Sehr oft machte uns die Patientin auf eine bestimmte Stelle aufmerksam, an der wir dann, während zuerst nichts zu fühlen war, genau ihrer Voraussage entsprechend, in den nächsten Tagen ganz in der Tiefe ein kleines Knötchen entdeckten, das dann seinen typischen zyklischen Verlauf durchmachte. Diese Tatsachen zusammen mit dem schub- 
weisen Auftreten mehrerer Knötchen auf einmal sprechen u. E. mit zwingender Notwendigkeit für die Annahme der hämatogenen embolischen Entstehung dieser Knoten. Um einen strikten positiven Beweis dafür zu erbringen, hätten wir uns zur Excision einer ganz beginnenden Effloreszenz, vielleicht noch während oder unmittelbar nach dem oben beschriebenen Schmerzstadium, entschließen müssen. Eine solche war uns leider nicht möglich. Immerhin werden wir soviel sagen können, dab unsere $h$ istologischen Ergebnisse zum mindesten unserer Annahme nicht entgegen stehen: Die Knoten b) und $d$ ) scheiden wir fuir die Beurteilung dieser Frage von vornherein aus, auch Knoten a) zeigt keinen nach dieser Richtung hin zu verwertenden Befund. Anders Knoten c). Hier finden sich sowohI die beiden genau ihrem Sitze nach beschriebenen Tuberkel-Konglomerate, als auch das tuberkulöse Granulationsgewebe, welches sich in der Adrentitia der Vene entwickelt hat, grade an solchen Stellen, welche in den vorhergehenden Schnitten durch besonders reichliche Gefäßentwicklung ausgezeichnet waren. Fügen wir hinzu, daß, wie schon oben gesagt, in den Tuberkeln selbst Gefäßreste eine Zeitlang nachweisbar waren, so werden wir immerhin, besonders wenn man sich an die von Wechsberg gefundene hochbedeutsame Tatsache des außerordentlich schnellen Schwindens der Gefäßstruktur bei von Tuberkelbazillen invadierten Gefäßen erinnert, mit einer gewissen Berechtigung diese Befunde in unserem Sinne deuten dürfen. Wie gesagt, absolut beweisend sind sie nicht. Beweisend sind für uns nur die klinischen Tatsachen.

Es dürfte hier der Ort sein, auf Philippsons ${ }^{1}$ ) Arbeit „Über Embolie und Metastase in der Haut" einzugehen. $\mathrm{Ph}$. meint, daß den Venen der Haut eine besondere Disposition, auf Entzündungsreize zu reagieren, zukomme und daß die hämatogene auf Embolie von Mikroorganismen zurückzuführende Entzündung der Haut, insbesondere des Unterhautzellgewebes nicht wie sonst an den kleinen Arterien and Kapillaren, sondern an den $V$ enen einsetze. Zum Beweise für seine Ansicht führt er die auf akuter lepröser Venenentzündung beruhenden Erythemausbrüche beim Aussatz an, ferner die Befunde von primärer Venenveränderung bei auf dem Blutwege entstandenen Syphilomen der Früh- und Spät-Periode und schließlich auch einen Fall von Folliklis oder wie er es nennt „Dermatitis nodularis necrotisans". In diesem sowohl als auch in einem zweiten (Archiv f. Derm. Bd. LV) publizierten Falle dieser Affektion fand $\mathrm{Ph}$. eine primäre $\mathrm{Phlebitis}$ einer rel. großen Vene des Unterhautbindegewebes mit Thrombose derselben und nachfolgender $\mathrm{Ne}$ krose, der sowohl der Inhalt des Gefäßes, als auch seine Wand und das

1) Archiv f. Dermatol. Bd. LI. 
umgebende Riesenzellen enthaltende Infiltrat anheimfielen. Es gelang ihm außerdem bei zwei Knötchen der tinktorielle Nachweis von Tuberkelbazillen und die positive Überimpfung auf ein Kaninchenauge. Er schließt aus diesen Befunden, daß nicht nur die Arterien, sondern auch - und dies soll das bei weitem häufigere sein - die Venen des Uuterhautbindegewebes der primäre Angriffspunkt der durch Embolie in die Haut verschleppten Bazillen sei. Diese Auffassung teilt auch Törö $\mathbf{k}_{.}{ }^{\mathbf{1}}$ ) Er hat in 2 Fällen je ein in der Lederhaut sitzendes Knötchen untersucht, an welchem makroskop. noch keine Zeichen von Nekrose wahrnehmbar waren. Im ersten Knötchen fand er eine Endophlebitis nebst perivaskulären Infiltraten, im zweiten eine thrombosierte Vene, deren Seitenäste ebenfalls zum Teil thrombosiert waren und die umgeben war von einem Streifen nekrotischen Bindegewebes. Je eine benachbarte Arterie und Vene zeigten ausgedehnte perivaskuläre Infiltrate, ebenso auch die kleinen Äste derselben. Noch weiter vorgeschrittene Veränderungen derselben fand er in einem ihm von Dubreuilh überlassenen Knötchen: In der Cutis einen krümlichen nelsot. streifenförmigen Herd, in dem verstopfte Gefäße deutlich nachgewiesen werden konnten, nach den Seiten und nach unten war ein diffuses Infiltrat vorhanden, welches sich gegen das subkutane Gewebe scharf abgrenzte. Unsere eigenen früheren bistologischen Ergebnisse (D. Archiv f. klin. Med. Bd. LXXI, pag. 587 ff.) sind für die Entscheidung dieser Frage unerheblich. Dagegen hatte $\mathrm{Juliusberg^{2 }}$ ) folgenden wichtigen Befund: Knötchen a): Ein dichtes Zellinfiltrat im subkutanen Gewebe bestehend aus einkernigen Leukocyten und gewucherten Bindegewebszellen; im Zentrum eine obliterierte große Arterie, in einem Teil ihres Verlaufes nekrotisch und von nekrotischen Massen umgeben. Eingestreut in das Infiltrat reichliche typische Langhanssche Riesenzellen. Knötchen $\beta$ ): Diffuse aus mononucleären Leukocyten bestehende keine Riesenzellen enthaltende Infiltrate, im Zentrum derselben eine thrombosierte Vene. Die äbrigen Befunde Juliusbergs sind ancharakteristisch. Sehr bestimmte Angaben dagegen macht Pinkus: (ebenda pag. 223). Die Effloreszenz der Folliklis besteht in einer eigentümlichen zentralen Nekrose, welche lange Zeit einen Teil des Gewebes, namentlich die elastischen Fasern, verschont, und aus einem peripheren Saum circumvasculärer Infiltration. In histogenetischer Auffassung deckt sich dieser Befund am ehesten mit den Folgen von Thrombose und periphlebit. Veränderungen.

Alle diese Autoren - Philippson, Török, Pinkus, Julius berg - sehen also als das Primäre bei der Histogenese der Follikliseffloreszenz eine Thrombose einer großen subkutanen Vene an,

1) Mraceks Handbuch der Hautkrankheiten, Artikel „Dermatitis nodularis necrot." pag. 446 ff. und Archiv f. Dermat. Bd. LVIII.

2) VII. Kongreß der deutschen derm. Gesellschaft. Breslau 1901. Teil. I, pag. 216 ff. 
Juliusberg außerdem noch in einem Falle die einer Arterie; sie glauben demnach - und Philipps on begründet dies auch ausführiich - daß der Bazillen-Embolus durch die kleinen und kleinsten Arterien, die arteriellen und venösen Kapillaren, sowie die kleinen Venen der Haut hindurchgehe, um dann, wenn alle diese Hindernisse passiert sind, in einer rel. großen Vene des Unterhautbindegewebes - denn um eine solche handelt es sich immer - nachträglich stecken za bleiben und lokale Verändierungen zu erzeugen, Diese Auffassung des ganzen $\mathrm{Her-}$ ganges erscheint uns, obwohl durch Phs. Autorität gestützt, doch etwas gezwungen, hauptsächlich deswegen, weil sie von den Tatsachen, welche wir sonst in der allgemeinen Pathologie zu sehen gewohnt sind, gänzlich abweicht. Wir müßten dann eben annehmen, daß die Arterie (Fig. 1) in unserem Knoten $a$, die Vene mit dem organisierten Thrombus in unserem Knoten $c$ (Fig. 3), sowie die thrombosierte Arterie und Vene in $J$ u li u s bergs Knoten $\alpha$ und $\beta$, und die thrombosierten Venen der anderen obengenannten Autoren die primäre, durch den Bazillen-Embolus hervorgerufene Läsion sind. Diese Vorstellung, daß ein doch relativ sehr kleiner Bazillen-Embolus einmal in einer Arterie haften bleibt, ein andermal bis in eine relativ große Vene vordringt, scheint uns eine so einschneidende Revision unserer allgemeinen pathologisehen Anschaunngen vorauszusetzen, daß wir uns vorläufig nicht zu ihr bekennen möchten.

Vielleicht ist übrigens die Kluft zwischen unserer und $\mathrm{Ph}$. Auffassung gar nicht so unüberbrückbar, wie es scheint. Wäre es nicht möglich - wir deuteten dies bereits oben an - daß in den Fällen, wo die Vene scheinbar der locus primae affectionis ist, der Embolus auf dem Wege der arteriellen vasa vasorum ${ }^{1}$ ) in die Wand und von da durch den Saftstrom zur Intima der Vene gelangt ist? Und ist diese Deutung, dje die ganze Differenz mit einem Schlage lösen würde, nicht eine ganz verständliche - nicht nur eine bestechliche?!

Die Beurteilung des Charakters des zweiten Bestandteiles der Folliklis.Effloreszenz, des Infiltrates bietet in gleicher Weise große Schwierigkeiten. Ten o es on, Lered de und Martinet (Aunales de Dermat. 1896, pag. 913) beschreiben verkäste Herde, und an der Peripherie von deren jüngeren Stadien ein Zellinfiltrat aus fixen und lymphoiden Zellen, an einer Stelle auch eine Riesenzelle. Sie sprechen selbst ihren Befund als infektiöses Granulom an. Hallopea und Bureau (Annales de Dermat. 1896, pag. 1310) fanden in der Gegend der Schweißdrüsen Plasmazellen und epitheloide Zellen, in späteren Stadien auch Riesenzellen und Entzündung einer großen Vene. Veillon ${ }^{2}$ )

1) Für die nodösen, klinisch der Folliclis sehr ähnlichen Syphilide der Sekundärperiode ist diese Rolle der vasa vasorum bereits von Marcuse (Archiv f. Derm. Bd. LXIII. pag. 14 und 15) besprochen worden, desgleichen von E. Nei sser (D. med. W, 1903. Nr. 37) für die syphilit. Phlebitis.

2) In Beauprez: Contribution à l'étude de la folliclis. Thèse de Paris 1898. Ref. Annales de Dermat. pag. 814 von 1898. 
spricht ebenfalls von einer diffusen Infiltration, in welcher einige $\mathrm{nekro}$. tis che Herd e nachweisbar waren. Keine Bazillen. Tierversuche negativ.

Wir gehen wohl nicht fehl, wenn wir sagen, daß die soeben geschilderten Befunde so manches haben, was für ihre tuberkulös e Natur spricht, sicherlich nichts, was dagegen za verwerten wäre.

Ganz unzweifelhafte tuberkulöse Veränderungen beschrieben Macleod und Ormbsy ${ }^{1}$ ) aus Fox K Klinik. Es handelt sich um eine 25jährige Frau mit Knoten an den Unterschenkeln und ein 17monatliches Kind mit spina ventosa und suspecten Lungen; beide Extremitäten wiesen an verschiedenen Stellen harte tiefgelegene erbsen- bis haselnußgroße Knoten auf. Im ersten Falle fanden die Verfasser Endo- und Peripblebit is des Hypoderms, sowie ein starkes lymphocytäres Infiltrat, in der $\mathrm{Cu}$ tis ein sehr massiges Infiltrat, im Zentrum desselben Nekrose, Bindegewebs- und elastische Fasern zerstört, nach der Epidermis zu ty $\mathrm{p}$ is che Tuberke l. Keine Bazillen. Meerschweinchen-Impfung negativ; im 2. Fall, von dem 4 Knoten herausgeschnitten wurden, war PhIe biti s des Hypoderms vorhanden, in der Cutis ein sehr dichtes Infiltrat, Bdg.und elast. Fasern zerstört, viele Riesenzellen, in einer 2 Bazillen. Beide Fälle sind als tuberkulös aufzufassen. Von den bereits erwähnten Autoren spricht $\mathrm{Ph}$. ansdrücklich davon, daß seine Infiltrate Riesenzellen enthalten und bildet solche auch ab, dazu kommt, daB er Tb.-Bazillen nachwies und ein Kaninchenauge mit positivem Erfolg impfte. Wir werden also an der tuberk. Natur des Infiltrates in keiner Weise zweifeln können, wenn dieselbe auch, rein histologisch betrachtet, nicht so überzeugend im mikroskopischen Bilde erscheint. Juliusberg, dessen Präparate ebenfalls oben ausführlich geschildert sind, sagt zwar (1. c.) "meine Befunde haben durchaus nichts für Tuberkulose charakteristisches, da erstens die Elemente des Tuberkels nicht vollständig vorhanden sind (Fehlen der epitheloiden Zellen) und zweitens auch die Anordnung der Zellen nicht der des Tuberkels entspricht"; wir können jedoch seine Einwände als stichhaltig nicht anerkennen. Das Fehlen der epitheloiden Zellen in einem Riesenzellen führenden Infiltrat berechtigt nicht ohne weiteres, die nicht tuberkulöse Genese dieses Infiltrats zu statuieren. Es kann sehr wohl dadurch erklärt werden, das erstere von mononucleären Leukocyten überwuchert worden sind. ${ }^{*}$ ) Noch weniger Wert möchten wir in Js. Falle der Anordnung der Zellen beilegen. Auch das tuberkulöse Granulationsgewebe läbt eine solche regelmäßige Anordnung der Elemente des Tuberkels, wie sie J. fordert, vermissen. Es wird niemand daran denken, der von Unna "Lupus diffusus" (Histopath. d. Hautkrankh.) genannten Varietät dieser Krankheit ihren tuberkulösen Charakter deshalb abzusprechen, weil sie

1) Report on the histopathology of two cases of cut. tuberculides, in one of which tubercle-bacilli were found. (Brit. Journal of Dermatol. Oktober 1901. Ref. nach Annales de Dermat. 1902, pag. 824.)

2) Ba umgarten. Über die pathol.-histolog. Wirkung des Tb.Bazillus. (B. klin. Wochenschrift 1901, Nr. 44, pag. 1109.) 
keine circumscripten Tu berkel, sondern ein diffuses, den Gefäßen folgendes Infiltrat zum anat. Substrat hat. Für die Törökschen Fälle läßt sich zwar die tuberkulöse Natur der Knoten nicht beweisen, aber auch hier erscheint sie in hohem Grade wahrscheinlich. Die beschriebenen Infiltrate sprechen zum mindesten nicht gegen, die regressiven und nekrotischen Stellen in den Schnitten dagegen sehr für Tuberkulose. Pinkus schreibt nichts genaueres über die Beschaffenheit des Infiltrates wir müssen uns daher ein Eingehen auf seine Befunde versagen.

Wenn wir zusammenfassen, so glauben wir nicht fehlzugehen, wenn wir, mit Ausnahme der Pinkusschen Fälle, die nichtganzsichersind, inallen bishergenannten Fällen eine aufembolischem Wege entstandene Tuberkulose mit mehr oder minder großer Sicherheit annehmen.

II. Gruppe. Dem gegenüber darf nun aber nicht vergessen werden, daß außer diesen besprochenen Fällen immer noch ein erheblicher Rest übrig ist, der nur rein entzündliche Veränderungen aufzuweisen hat. Hierher gebört ein Teil von unseren l. c. beschriebenen Typen, ${ }^{1}$ ) sowie auch teilweise Juliusbergs Fälle und unsere Fälle II und III und viele aus der Literatur, die wir in Töröks Arbeit (s. oben) aufgezählt finden. (Überhaupt möchten wir an dieser Stelle bemerken, daß wir durchaus nicht alle Literaturangaben in den Bereich unserer Erörterung ziehen resp. gezogen haben, sondern nur diejenigen, welche etwas nach irgend einer Richtung hin Charakteristisches bringen.) Philippson hat gemeint, daß diese Differenzen in den Befunden der Autoren sich daraus erklären, daß die Effloreszenzen einmal in verschiedenen Stadien ihrer Entwicklung und ferner in verschiedener Tiefe, id est häufig nicht tief genug excidiert worden seien. Zum Teil mag diese Erklärung zutreffen, aber doch nicht für alle Fälle. Es ist uns aufgefallen, daß die eben in Rede stehenden Knötchen Krankheitstypen angehören, die klinisch der sogenannten oberflächlichen Folliklisform zuzurechnen sind, und es will uns scheinen, daß diese klinisch als zu Recht bestehende Scheidung in oberflächliche und tiefe Formen auch histologisch zum Ausdruck kommt und zwar dadurch, daß die oberfläehlichen Knötchen nur uncharakteristische perivaskuläre diffuse Infiltrate aufweisen. Es erscheint unmöglich, eine Begründnng dieser Verhältnisse zu geben, es bleiben nur Vermutungen. Vielleicht sind die Lebensbedingungen für die Bazillen, wenn sie in die straffe Cutis kommen, andere, als wenn sie in den in lockerer Umgebung liegenden Gefäßen des Unterhautbinde-

1) Cfr. außerdem VII. Kongreß der d. dermat, Gesellsch. Breslau 1901. I. Teil. pag. 209 
gewebes sich deponieren, vielleicht ist auch der Umstand von Bedeutung, daß infolge der oberflächlicheren Lage der Knötchen eine sekundäre Kokkeninfektion von außen leichter Eingang findet und die histolog. Struktur beeinflußt.

Wie dem auch sein mag, vorläufig müssen wir, bis die Frage definitiv geklärt ist, zwei Typen unterscheiden: den tiefen mit tuberkulösen Veränderungen, den oberflächlichen mit diffusen entzündlichen Infiltraten resp. Thrombophlebitiden. Beiden gemeinsam ist aber unzweifelhaft ihre Entstehung auf embolischem Wege und zwar, wie wir wohl mit Sicherheit annehmen dürfen, durch den Tuberkelbazillus selbst.

Setzen wir dies als tatsächlich voraus, so entstehen sofort eine Anzahl anderer Fragen: Warum sind unsere Bazillenbefunde in diesen so eindeutig erscheinenden tuberkulösen Produkten so außerordentlich spärlich, daß man sie fast als negativ bezeichnen möchte? Warum haben wir trotz zahlreicher Versuche und fleißiger Arbeit nur so wenige positive Impfversuche aufzuweisen? Schließlich warum zeigen unsere Folliklis-Patienten nicht zahlreichere Herde visceraler Tuberkulose, warum sterben nicht viele von ihnen bei einem größeren Schube an akuter Miliartuberkulose? Den bazillären Überschwemmungen stehen ja doch die inneren Organe (Gehirn, Leber, Nieren etc.) ganz ebenso offen wie die Hautgebiete?

Wir haben für die ersten beiden Fragen in einer früheren Arbeit (cf. 1. c.) in der Annahme abgeschwächter Tu berkelbazillen ${ }^{1}$ ) eine Erklärung und Deutung gesucht, die wir auch heute noch festhalten, so sehr wir uns der vielen Angriffspunkte, die sie bietet, bewußt sind. Es ist wenigstens der Versuch einer Erklärung bis zu einer besseren und klareren Erkenntnis. Auch für die dritte Frage müssen wir eine eigentliche Antwort schuldig bleiben. Vielleicht spielt auch hier die hypothetische Abschwächung der Tuberkelbazillen eine Rolle, vielleicht wirken die Schutzkräfte, über die der Körper in so reichem Maße den Tuberkelbazillen gegenüber verfügt, in den blutreichen parenchymatösen Organen so energisch, daß sie die Bazillen gar nicht zur Entwicklung und Vermehrung kommen lassen. Gerade für diese letztere Vermutung würde

J) Diese Möglichkeit wurde bereits 1899, wie wir nachträglich erfahren haben, von $\mathrm{J}$ a d a s $\mathrm{s}$ o h $\mathbf{n}$ in seinem Vortrage „Die tuberkulösen Erkrankungen der Haut" (B. klin. W. 1899. pag. 1013) erörtert. 
die uns allen ja längst bekannte Tatsache der relativen Im m u nität gewisser Organe (Herz, Muskulatur, Schilddrüse etc.) dem Tuberkelbazillus gegenüber sprechen. Ganz sicher tragen auch äußere auf die Haut einwirkende Schädlichkeiten irgend welcher Beschaffenheit, denen diese fortwährend ausgesetzt ist, dazu bei, hier einen locus minoris resistentiae zu schaffen und die Ansiedlung der Bazillen zu begünstigen (l. c. pag. 595). Und noch eine vierte Frage liegt nahe. Warum ist die Folliklis trotz des ungeheuren Heeres von tuberkulösen Erkrankungen jeder Art eine so relativ seltene Affektion? Ist sie es wirklich, oder scheint es nur so? So sicher es ist, daß eine große Anzahl von Folliklisfällen unerkannt bleiben (cf. Fall V und VI) gerade aus der Privatpraxis, so ist doch immer die große Seltenheit der Affektion im höchsten Grade auffallend. Eine Erklärung dieser Tatsache vermögen wir nicht zu geben; auch eine Hypothese versagen wir uns.

In wie weite Gebiete von Spekulation und Phantasie Hypothesen bei diesen Fragen führen, zeigt uns Hall o pea u, ${ }^{1}$ ) den wir zum Schluß zitieren. H. nimmt für die Fälle, in denen es zur Eruption von Tuberkuliden kommt, eine Art besonderer Reaktion der Haut auf das tuberkulöse Virus an; diese Reaktion ist, wie man aus der Verschiedenheit der Formen ersieht, eine sehr variable und soll auf der variierenden chemischen Konstitution der Gewebe beruhen. Was für eine besondere Modifikation der chemischen Zusammensetzung vorliegt, darauf bleibt allerdings $\mathrm{H}$. die Antwort schuldig.

\section{Nachtrag.}

Vorstehende Zeilen waren bereits druckfertig, als Klingmüllers Arbeit „Zur Wirkung abgetöteter Tuberkelbazillen und der Toxine der Tuberkelbazillen" (Berl. klin. W.Nr. 34) vom 24. Aug. 1903 erschien. K. geht aus von der Tatsache, daß die Injektionsstellen unserer diagnostischen Tuberkulin-Einspritzungen nicht ganz reaktionslos bleiben und daß ferner solche alte Injektionsstellen auf erneute Tuberkulin-Injektionen, welche an irgend welchen anderen Stellen des Körpers gemacht werden, eine örtliche Reaktion zeigen. Die Richtigkeit dieser beiden Beobachtungen können wir auf Grund unserer eigenen Erfahrungen an einem ziemlich ausgedehnten Material durchaus bestätigen. Klingmüll er exzidierte einzelne solcher reagierender Reaktionsstelleu und fand bei der histologischen Untersuchung zahlreiche größere und kleinere Herde lupöiden Charakters (zentral epitheloide and Riesenzellen, peripher Infiltrationszellen) im subkutanen Gewebe. Seine weiteren experimentellen Untersuchungen hatten das Resultat: $\mathrm{DaB}$ a) das Alttuberkulin abgetötete, jedoch noch virulente, $d$. h. toxinhaltige Bazillen enthalte, $b$ ) da $\beta$ das Tuberkulin auch nach Entfernung dieser Bazillen durch ein Reichel-Tonfilter dieselben Tuberkulose ähnlichen Veränderungen im Gewebe erzeugt, d. h.

1) Hallopeau. Sur une forme bulleuse des toxituberculides (Annales de dermat. 1908, pag. 35 ). 
daß die Toxine des Tuberkelbazillus anatomische Tuberkel hervorzurufen im stande sind. Diese letztere Beobachtung entspricht durchaus den Untersuchungen Sternbergs $\mathbf{s}^{1}$ ) die dieser vor etwa dreiviertel Jahren zur Entscheidung variierender Literatur-Angaben ïber dieses Thema publiziert hat.

St. hat, allerdings auf anderem Wege als $\mathrm{K}$., den Nachweis geführt, daB tote Tuberkelbazillen im Tierkörper dieselben Veränderungen hervorrufen können, wie lebende. Sie erzeugen, in entsprechend großer Menge injiziert, Knötchen, die aus epitkeloiden und Riesenzellen bestehen und auch $a b$ und zu verkäsen, in geringerer Menge bewirken sie bei dem Versuchstier einen Marasmus, der in verschieden langer Zeit zum Tode führt. Die pathologische Wirksamkeit des Tuberkelbazillus ist mithin an eine dem Bazillenleib anhaftende Substanz gebunden, mit anderen Worten: die Toxine des Tuberkelbazillus sind im stande, Tuberkel zu erzeugen.

Wir möchten an dieser Stelle nachbolen, dab wir Klingmüllers Beobachtungen, soweit sie sich auf die histologische Beschaffenheit reagierender Injektionsstellen beziehen, durchaus bestätigen können. Auch wir haben - vorläufig allerdings nur in einem Falle - eine solche Stelle. nebst einem Stück des umgebenden gesunden Gewebes excidiert: Im letzteren fanden sich rein entzündliche Veränderungen, im ersteren war die untere Cutis der Sitz zahlreicher, mehr oder minder circumscripter, ebenso wie bei K. an die Gefäße sich anlehnender Epitheloid-Zellen.Tuberkel, deren Rand einkernige Zellen bildeten. Auch in der Tiefe des im übrigen normalen Unterhautfettgewebes fanden sich an mehreren Stellen umschriebene Tuberkel. Spärliche Langhanssche Riesenzellen waren nackweisbar. Die betreffenden Untersuchungen sind von Herrn Dr. Ortmann ausgeführt, dem ich für Überlassnng der Resultate zu Dank verpflichtet bin.

Die Konsequenzen der Untersuchungen der beiden oben genannten Autoren sind zweiffellos von der größten Wichtigkeit für die Pathogenese der Tuberkulide. Ist es richtig, daß die Toxine des Tuberkelbazillus Tuberkel, ja, sogar verkäsende Tuberkel erzengen können, so erscheinen natürlich sofort unsere negativen Impfungen und die Spärlichkeit der Bazillenbefunde in einem ganz anderen Lichte: Sie sind dann eine Bestätigung der Annahme der Toxin-Natur unserer Affektion. Indessen soweit möchten wir doch vorläufig nicht gehen. Einstweilen tragen wir und trugen wir bereits damals vor bereits dreiviertel Jahren Bedenken, Sternbergs L aboratoriumsversuche so ohne Weiteres auf den Menschen zu übertragen. St. sagt ausdrücklich, daß eine sehr große M enge toter Tuberkelbazillen notwendig ist, um die in Rede stehenden tuberkuloseähnlichen Veränderungen zu erzeugen. Es erscheint uns im hohen Grade fraglich, ob unsere FolliclisKranken wirklich so erhebliche Mengen Toxine produzieren, wie sie offenbar zur Erzeugung von Folliclis-Knoten notwendig sein müßten; wir meinen, der Allgemeinzustand derartiger

1) Sternberg, Experimentelle Unters. über die Wirkg. toter Tuberkelbazillen. Zentralbl. für allgem. Pathol. u. path. Anat. 1902. Band XIII. $\mathrm{Nr} .19$. 
Patienten müßte bei weitem schlechter sein, als ein großer Teil der in Betracht kommenden Patienten ihn tatsächlich darbietet.

Einen ähnlichen Einwand könnte man den Folgerungen gegenüber machen, die sich aus Klingmüllers Untersuchungen zu ergeben scheinen. Da wir über die Toxinmenge, die ein 'Tuberkulose-Kranker, z. B. mit einer LungenTuberkulose, in der Zeiteinheit produziert, und in den Kreislauf hinein schickt. noch gar nicht unterrichtet sind, und ebenso wenig Klarheit besitzen über das Verhältnis dieser zu den in unseren diagnostischen Tuberkulin-Injektionen enthaltenen Toxin-Mengen, so scheint uns auch hier die Frage sehr diskutabel: sind denn in Praxi die von unseren Kranken produzierten Toxinwerte so erheblich, daß durch sie die Folliclis-Effloreszenzen erzeugt werden können?

Sei dem wie ihm wolle, soviel steht fest, daß die Toxintheorie der Folliclis jetzt einen gesicherten Hintergrund hat, während sie früher völlig in der Luft schwebte, und daß es weiterer ausgedehnter Untersuchungen bedürfen wird, um diese ganze Frage endgültig zu entscheiden.

Eine Art mittlerer Linie zwischen der bazillären und der Toxintheorie könnten vielleicht folgende Erwägungen bieten, die ich einer Erörterung mit Herrn Dr. Lubowski, Assistent der med. Univers. - Poliklinik entnehme: Es ist bekannt, daß die ersten Tuberkulin-Injektionsstellen bei erneuter TuberkulinInjektion viel stärkere Injektionserscheinungen aufweisen als das erste Ma!. Diese Tatsache läßt sich nur durch eine dadurch erworbene erhöhte Reizbarkeit dieses Gewebes erklären. Eine ebensolche Reizbarkeit mögen nun auch solche Stellen des Körpers haben, an welche vor einiger Zeit lebende oder tote Tuberkelbazillen embolisch verschleppt worden sind. Auch ohne daß dort histologische Veränderungen stattgefunden haben (durch Phagocytose etc. ist es nicht zur Tuberkelbildung gekommen), haben die Zellen eine gewisse inmunisatorische Überempfindlichkeit, id est Reaktionsfähigkeit für Tb.-Toxine davongetragen. Gelangen nun aus irgend einem tuberkulösen Herde Toxine in den Kreislauf, so reagieren diese Stellen ana$\log$ den Tuberkulin-Reaiktionsstellen genau wie ein tuberkulöser Herd, d. h. es kommt zur Bildung von Folliclis-Effloreszenzen oder sonstigen Tuberkuliden.

Vielleicht ist auch der Lichen scrofulosorum, den wir in Deutschland bisher fast allgemein den Haut-Tuberkulosen zurechneten, in diesem Sinne aufzufassen. Wir sehen so oft Fälle von dieser Affelstion, die klinisch fast gar keine Erscheinungen machen und erst auf eine Tuberkulin-Injektiond.h. dadurch daß sie reagieren, deutlich als Lichen 
skrofulosorum zur Kognition kommen. In diesen Fällen produziert also - die Richtigkeit unserer Theorie vorausgesetzt - der Körper nicht genügend Toxine, um die supponierten, überempfindlich immunisierten Stellen zur Reaktion d. h. zum deutlichen Hervortreten zu bringen, und erst unsere Tuberkulin-Injektion vermag dieses Minus an Toxinen soweit auszugleichen, daß eıne Reaktion eintritt. In anderen Fällen ist diese Zufuhr von Toxinen nicht notwendig, der Körper selbst produziert genug davon, um die betreffenden Stellen zur Reaktion zu bringen: Der betreffende Patient hat einen klinisch unzweifelhaften deutlichen Lichen scrofulosorum schon vor der Tuberkulin-Injektion.

Für diese Auffassung scheint uns auch folgende Erfahrung zu sprechen, die wir oft genug gemacht haben. Es kommt jemand mit einem ansgesprochenen Lichen scrofulosorum zur Behandlung, derselbe heilt unter allgemeinen hyg. Maßnahmen ab, der Patient wird entlassen, ohne daß an seiner Haut irgend etwas Pathologisches zu erkennen wäre. Nach kürzerer oder längerer Zeit ist er wieder da mit einer an denselben Stellen lokalisierten neuen Eruption von Lichen scrof. Fs ist nicht zu leugnen, daß für diese Fälle die rein bazilläre Theorie des Lichen scrof. etwas gezwungen erscheint: Gerade hier wird man eher zu der Annahme neigen, daß der betreffende Pat. bei seiner Aufnahme stark mit einem ausgesprochenen Lichen scrof. reagiert hat, daß diese Reaktion - während seines Aufenthaltes abgeklungen ist, und daß er nun, entlassen und schlechten hygienischen Bedingungen unterworfen, auf einen neuen Toxinschub wieder an den gleichen Stellen mit einem neuen Lichen scrof. reagiert.

\section{Literatur.}

Die im Text erwähnten Arbeiten sind nicht wieder angeführt. Über die ältere Literatur vergl. Török: Mrazeks Handhuch der Hautkrankheiten. p. 462. A lexand er: D. A. f. klin. Med. Bd. LXXI, p. 595. Motty, M. Toxituberculides des mains et des pieds. Annales de Derm. 1901. pag. 754.

Hallopeau, M. Sur un cas de tuberculides papulopustuleuses isolées et agminées avec infiltration en nappe. Annales de derm. 1901. p. 946. Castel. Tuberculides des organes genitaux. Annales de derm. 1901. pag. 71 .

Stra Bmann. Tuberkulide der Vulva. B. klin. W. 1901. pag. 615. Zollik ofer. Über die Hauttuberkulide. Korrespondenzblatt für Schweizer Ärzte. 1902. (Literatur.)

Delbanko. Ein Fall von Lupus erythematodes disseminatus B oeck. D. med. W. Nr. 36. 1903. Vereinsbeilage. pag. 282. 
Gaucher et Rostaine. Tuberculose cut. papuleuse. Annales de derm. Mai 1903.

A. Neis ser. Die tub. Hauterkrankungen. D. Klinik. X. Abt. 2. 1902.

\section{Literatur-Nachtrag (für die Arbeit selbst nicht mehr verwertet).}

Pautrier. Les Tuberculoses cut. atypiques (Tuberculides). Paris 1903. (Monographie mit ganz ausführlicher Literatur.)

Ni k o la u. Contrib. à l'étude des Tuberc. Ann. de derm. 1903, p. 713.

Moberg. Lupus eryth. dissem. (pap.-nekrot. Tuberculid). Stockholmer derm. Ges. Ref. Mon. für prakt. Derm. 1903. Band XXXVI. p. 451.

B eck und GroB. Über Lichen scrophul, und dessen Beziehungen zo den Tuberculides cut. Dari e r. Arch. f. Kinderheilk. Bd. XXXIV. p. 25.

Bruusgard. Beitrag zu den tub. Hauteruptionen (Erythrodermia exfol. univers, tuberculosa.) Archiv f. Dermat. Bd. LXVII. p. 227. Jordan. Über einen den Tuberculides acnéif. et necrot ähnlichen Krankbeitsfall. Mon. für prakt. Dermat. Bd. XXXVII. Nr. 12.

Graham Little. Krankenvorstellung e. Falles von pap.-nekrot. Tuberkuliden. Londoner derm. Ges. Ref. D. Zentralblatt Dez. 1903.

Sé e et Druelle. Tuberculides. Journal des malad. cut. et syph. Bd. XV. 1903. pag. 818.

\section{Erklärung der Abbildungen auf Taf. IV-VI.}

Fig. 1. Schnitt durch den Knoten a) von Fall I. Beginn der Serie. Zeiß. Okular. 2. Obj. A. A. Färbung nach Unna-Taenzer (s. Orceïn-Thionin). a) Intimaverdickung einer größeren Arterie, $b$ ) verdickte kleine Gefäße, c) tuberkulöses Granulationsgewebe, $d$ ) Epitheloidzellentuberkel mit beginnender Verkäsung, e) Riesenzellen, $f$ ) tuberkulöse Nekrose des Fettgewebes.

Fig. 2. Schnitt durch Knoten a) von Fall l. Ende der Serie. Zeiß. Okular 2. Obj. D. D. Färbung nach Unna-Taenzer (s. OrceïnThionin). a) Vollständige Endarteritis obliterans der sub a) in Fig. 2 gezeichneten Arterie. b) Tuberkulöses Granulationsgewebe (epitheloide Zellen und Leukocyten), das die Media und Adventitia der Art. völlig substituiert hat.

Fig. 3. Schnitt durch Knoten c) von Fall I. Beginn der Serie. Färbung mit Hämatoxylin - van Gies o n. Leitz. Oc. I. Obj. II. a) Vene, $b$ ) in Organisation begriffener Thrombus, c) normale Gefäße, d) Tuberkel mit Riesenzellen, e) tuberkulöses Granulationsgewebe in der Adrentitia einer anderen großen hier nicht sichtbaren Vene.

Fig, 4. Schnitt durch Knoten c) von Fall I. Mitte der Serie. Färbung: Hämatoxylin-Pikrinsäure. Leitz. Okular I. Obj. IV. a) Normales Fettgewebe, b) tuberkalöses Granulationsgewebe im Unterhautzellgewebe mit $a$ ) Riesenzellen, d) tuberkulös-nekrotisches Fettgewebe mit Kernbröckeln, e) durch den Härtungsprozeß ihres Inhaltes beraubte Fetträrume.

Fig. 5. Schnitt durch Knoten $b$ ) von Fall I. Färbung: W eigerts Elastinfärbung - van Gies on. Leitz. Okular. I. Obj. II. a) Epidermis, b) Cutis, des elastischen Gewebes fast völlig verlustig gegangen, c) Unterhautfettgewebe mit d) tuberkulösem frischem Granulationsgewebe, e) tuberkulös-nekrot. Fettgewebe, $f$ ) Endangitis tuberculosa (nur noch durch die Anordnung der elast. Fasern als Gefäß erkennbar) mit Riesenzellen, g) Endangitis tuberculosa caseosa. 


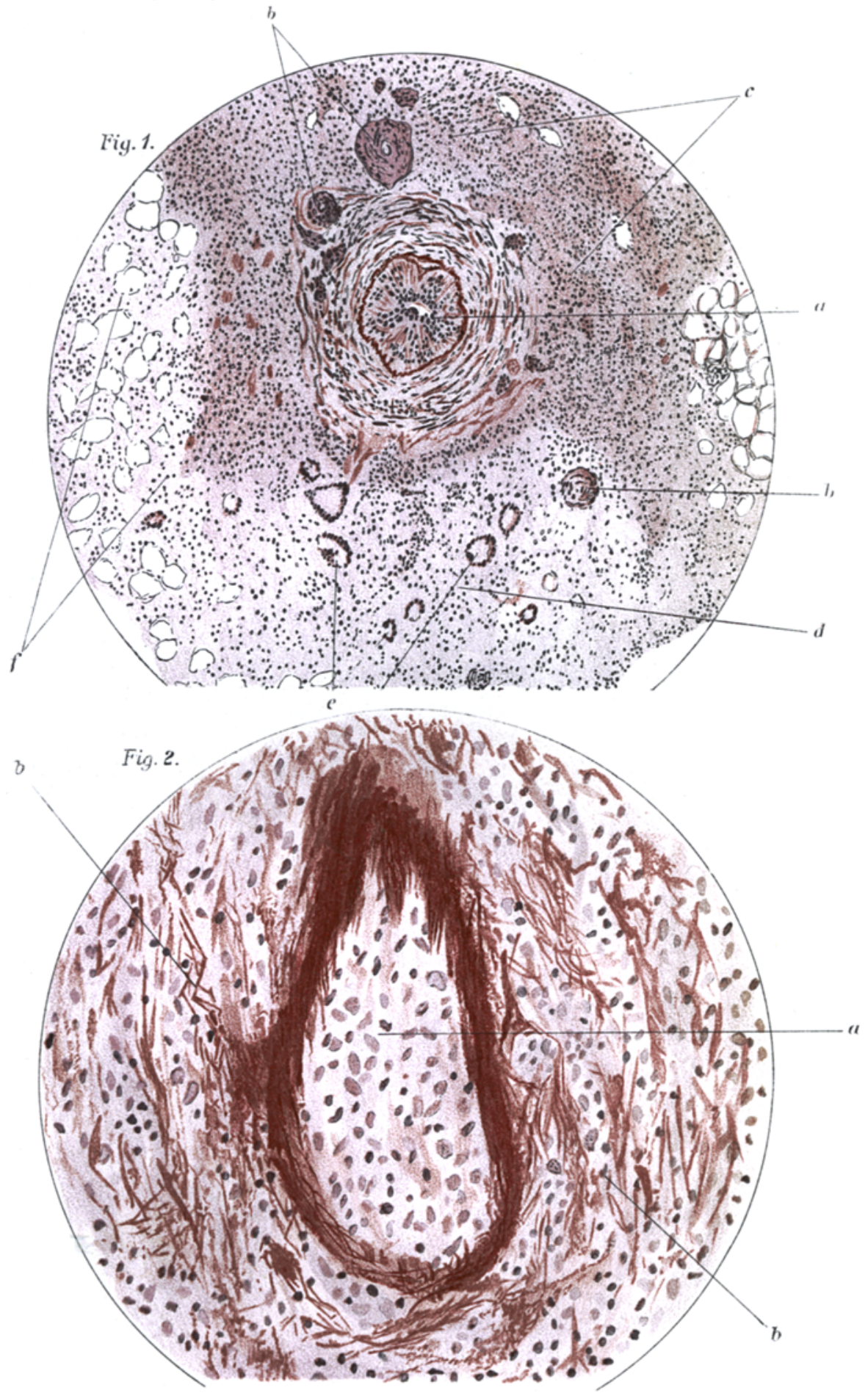




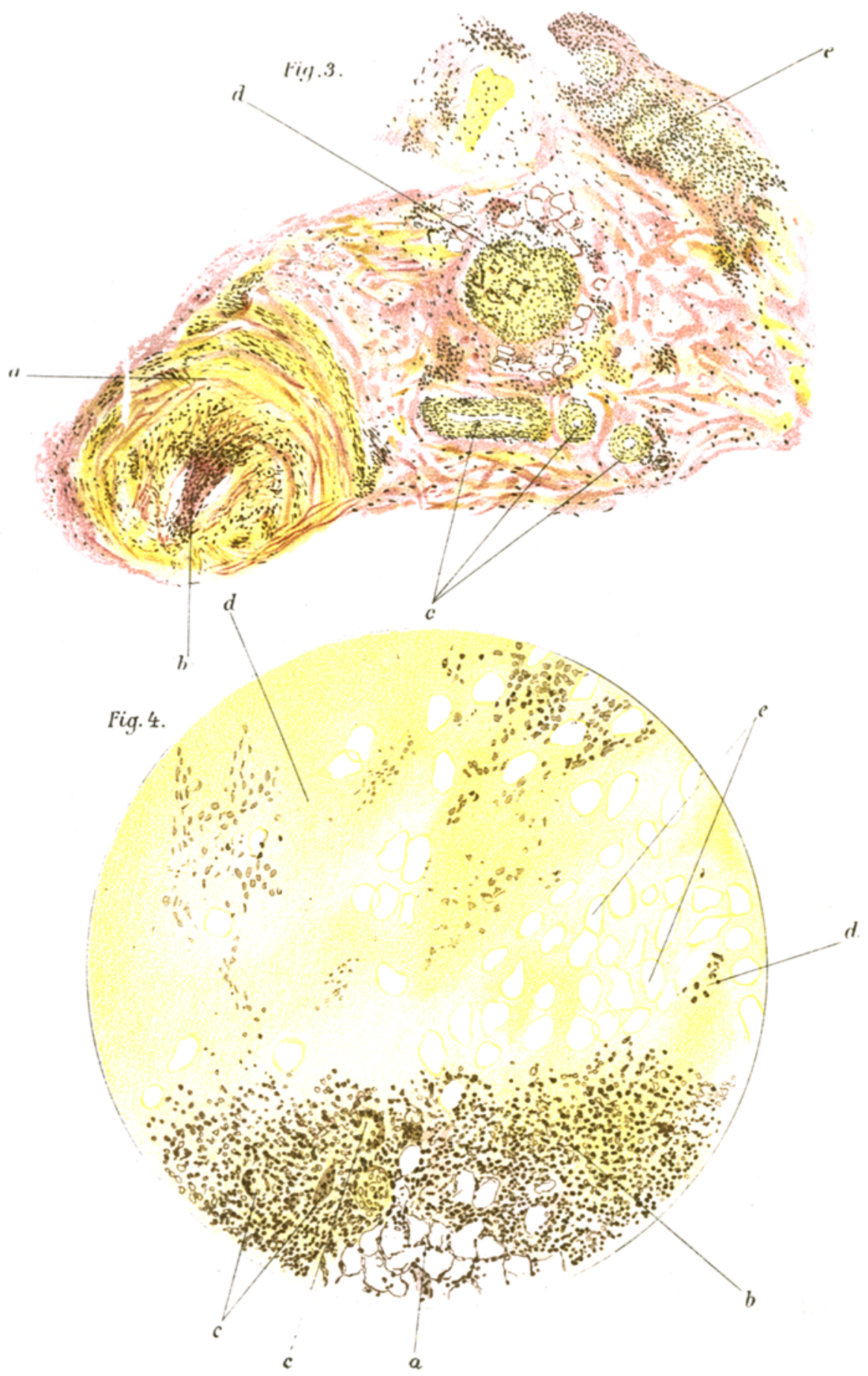

Alexander : Klinik u. Histologie der Folliclis. 


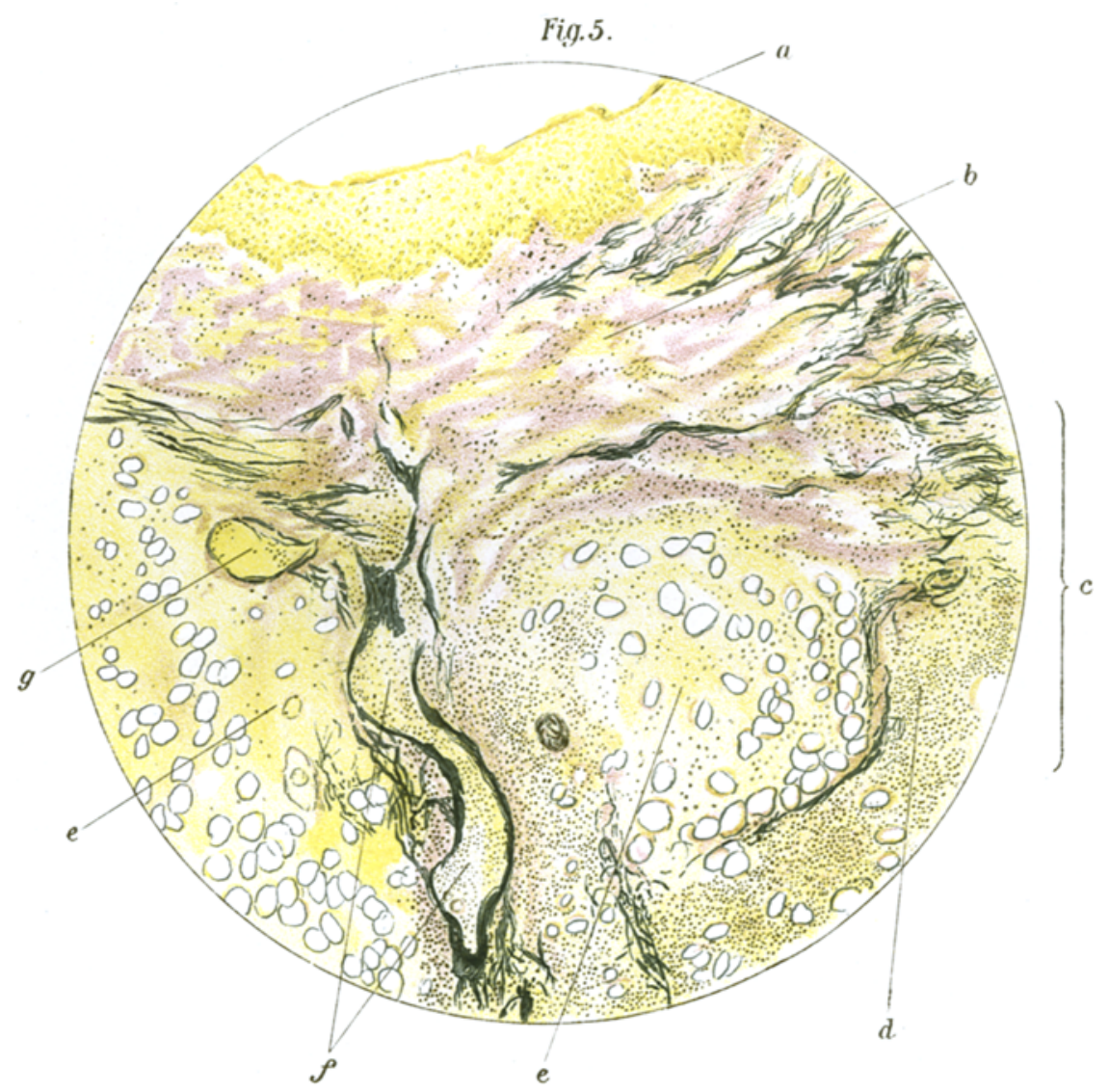

